\title{
Analysis of DNA curvature distribution in mycobacterial promoters using theoretical models
}

\author{
Rupali N. Kalate ${ }^{\mathrm{a}}$, B.D. Kulkarni ${ }^{\mathrm{a} *}$, V. Nagaraja ${ }^{\mathrm{b}}$ \\ ${ }^{a}$ Chemical Engineering Division, National Chemical Laboratory, Pune 411 008, India \\ ${ }^{\mathrm{b}}$ Microbiology and Cell Biology Department, Indian Institute of Science, Bangalore 012, India
}

\begin{abstract}
In this paper, 125 different mycobacterial promoters are analyzed for their DNA curvature distribution using several di- and tri-nucleotide dependent models of DNA curvature. Different models give similar behavior and therefore qualitative validation of the results. Mycobacterial promoters resembling the E. coli $\sigma^{70}$ type have almost $81 \%(85 \%)$ sequences having medium and high curvature profiles using dinucleotide-dependent models. Non- $E$. coli $\sigma^{70}$ type mycobacterial promoters have comparatively higher percent of low curvature profiles. Very few extended -10 promoters have low curvature profiles. Mycobacterial promoters having $\mathrm{A}_{n} T_{m}(n+m \geq 3)$ tract in the upstream region of -35 box and repeated in phase with each other have high curvature profiles. M. smegmatis promoters have high curvature profiles compared to M. tuberculosis promoters.
\end{abstract}

Keywords: Mycobacterial promoters; Transcription; Curvature models

\section{Introduction}

Transcription process in Mycobacteria may differ from E. coli and many other bacteria as mycobacterial genome has high $\mathrm{G}+\mathrm{C}$ content which affects codon usage and promoter recognition sites in an organism. Mycobacterial promoters like M. tuberculosis $65 \mathrm{kDa}$ [1], M. bovis BCG 64 $\mathrm{kDa}$ [2], and M. leprae $65 \mathrm{kDa}$ [3] are known to function in $E$. coli. However, mycobacterial promoters like M. tuberculosis 85A [4], recA [5] are known to be non-functional in E. coli. Thus, depending on the choice of expression host, myco-

*Corresponding author. Tel.: +91-20-589-3095; fax: +9120-589-3041.

E-mail address: bdk@ems.ncl.res.in (B.D. Kulkarni). bacterial promoters are classified as E. coli type and Non-E. coli type promoters. M. smegmatis and M. tuberculosis promoter analysis by Bashyam et al. [6] showed that occurrence of a TG motif near the -10 region is functionally significant for those having a non-functional -35 region. These promoters form a different class of promoters known as 'Extended -10 promoters'. The type of expression host, and the variation of the nucleotide sequence composition at the -35 and -10 regions of mycobacterial promoters [7] indicate that there exists immense variation in transcription initiation mechanism of mycobacterial promoters.

Transcription initiation is a multi-step, sequential process involving: (a) binding of RNA polymerase to the promoter leading to formation of a 
relatively weak closed initiation complex; (b) its isomerization to the more stable open complex that is accompanied by the separation of the DNA strands upstream and around the start site of the transcription; and (c) RNA polymerase escapes from the promoter after cycles of abortive initiation forming the stable elongation complex [8]. Promoter DNA undergoes drastic conformational changes during initiation of transcription. The necessary condition for open complex formation is that RNA polymerase must bind and bend the promoter DNA. This bending and subsequent torquing is responsible for melting the DNA and the formation of open complex $[9,10]$.

The role of DNA curvature has been studied extensively in E. coli [11-17]. The conformation of DNA is a function of its nucleotide sequence $[18,19]$. The three-dimensional structure of DNA is the effect caused largely by interactions between neighboring base-pairs [20-29]. Generally, periodic repetitions of curved DNA in phase with the helical pitch cause the DNA to assume a macroscopically curved structure. Several theoretical models for estimating DNA curvature from di- or trinucleotides have been devised, and require various types of experimental data [23,25,28-31]. It is to be noted, however, that these models are being debated for their generality [32]. The importance of DNA conformation in transcription initiation is, however, clear and it would be interesting to study the DNA curvature distribution within the mycobacterial promoters especially in view of the large variation in their transcription mechanism. The objective of this paper is to use six different di- and trinucleotide-dependent models of curvature prediction for analysis of mycobacterial promoters.

\section{System and methods}

\subsection{Data}

The data for curvature analysis was taken from compilation of mycobacterial promoters (see Appendix A, the original reference details of each entry can be obtained from authors upon request). This data set contain 125 different mycobacterial promoters, out of which 80 promoters have their transcription start site (TSS) mapped while the other 45 are the putative promoters. In the listed compilation, we have considered the sequence stretches between -50 and +10 bp with respect to the TSS for the promoters whose TSS is mapped. For the putative promoters, we have documented the sequence stretch between the 15bp upstream region of -35 box and $20 \mathrm{bp}$ downstream of the -10 box. The promoter sequence length varies from 34 to 71 nucleotides based on the availability of the nucleotide sequence upstream of the -35 region and downstream of the -10 region. In a few cases, for the same gene two or more different sequence frames are considered based on the alternate consensus probability. Thus, total 135 mycobacterial promoter sequences are used in this study.

\subsection{Curvature analysis}

For the purpose of analyzing curvature distribution within mycobacterial promoter sequences, we have used the following dinucleotide models based on: (i) experimentally determined wedge angles [25]; (ii) energy minimized values of roll and tilt angles [31,33]; (iii) X-ray crystallography of DNA oligomers [30]; and (iv) CalladineDickerson rules $[34,35]$. The trinucleotide models used include: (i) the model based on tabulation of preferred sequence locations on nucleosomes [23,28]; and (ii) DNase I cutting frequencies [29].

1. CURVATURE [36]: To obtain curvature map of each mycobacterial promoter, a window size of a $21 \mathrm{bp}$ nucleotide sequence is given as an input to the program and the curvature is obtained as an output. The results of this study are listed in Table 1 for each mycobacterial promoter. Various sub-groups of mycobacterial promoters are analyzed for nature of curvature profile and results are listed in Table 2.

2. De Santis et al. [33]: The curvature vector $\mathbf{C}$ $(n, v)$ representing, in the complex plane (in modulus and phase), the directional change of the double helix axis between sequence number $n$ and $n+v$ is calculated for each mycobacterial promoter sequence in the compilation. For this calculation, roll and tilt angle values (in 
Table 1

Nature of curvature profile for mycobacterial promoters using dinucleotide models based on (i) experimentally determined wedge angles, and (ii) energy minimized values of roll and tilt angles

\begin{tabular}{|c|c|}
\hline & Shipgelman et al. [36] ${ }^{\mathrm{a}}$ \\
\hline Low & $\begin{array}{l}\bullet \text { M. tuberculosis: T26, T119, T129, T130, } \\
\text { gyrA, cpn60, KatG } \mathrm{P}_{\mathrm{A}}\left(\mathrm{sp}^{\mathrm{c}}=19\right), \mathrm{KatG} \mathrm{P}_{\mathrm{A}}(\mathrm{sp}=15) \text {, } \\
\mathrm{KatG}_{\mathrm{C}}(\mathrm{sp}=22), \mathrm{KatG}_{\mathrm{C}}(\mathrm{sp}=14), \text { purC } \\
\bullet \text { M. leprae gro E1 } \\
\bullet \text { M. smegmatis: alrA, S5, S119, gyrB, ask, } \\
\text { rrnA P3 } \\
\bullet \text { M. paratuberculosis: pAJB303, } \\
\text { pAJB304, } \mathrm{P}_{\mathrm{AN}}, \mathrm{pAJB} 73 \\
\bullet \text { M. fortuitum repA }\end{array}$ \\
\hline
\end{tabular}

Medium $\quad \bullet$ M. tuberculosis: T3, T6, T80, T125, recA, rrnA P1, gyrB P3, 85A $\left(\mathrm{sp}^{\bullet}=17\right)$, $85 \mathrm{~A}(\mathrm{sp}=22), \operatorname{gyrB} \mathrm{P} 2, \operatorname{gln} \mathrm{A}(\mathrm{sp}=18)$, $\operatorname{gln} \mathrm{A}(\mathrm{sp}=10)$, KatG $\mathrm{P}_{\mathrm{B}}(\mathrm{sp}=20)$, KatG $\mathrm{P}_{\mathrm{B}}(\mathrm{sp}=22)$, purL, groE $(\mathrm{sp}=19)$, groE $(\mathrm{sp}=11), \operatorname{ahpC}$, $32 \mathrm{Kda}, 10 \mathrm{Kda}(\mathrm{sp}=17), 10 \mathrm{KDa}(\mathrm{sp}=15)$, $10 \mathrm{Kda}(\mathrm{sp}=8), 65 \mathrm{Kda}$, mpt64, metA, $38 \mathrm{Kda}, \mathrm{ppgK}$

-M. bovis BCG: hsp60 P2, ahpC, $23 \mathrm{~K}$, mpb 64,64 K

-M. leprae: $16 \mathrm{~S}$ rRNA, $28 \mathrm{Kda}, 36 \mathrm{~K}$, SOD -M. smegmatis: S4, S18, S21, S33, S65, S69, recA, acetamidase, rrnA P1, rrnA P2, rrnA PCL1, rpsL $(\mathrm{sp}=18), \mathrm{rpsL}(\mathrm{sp}=17)$, ahpC -M. paratuberculosis: pAJB86, pAJB125, pAJB300, pAJB305, pAJB301

-M. fortuitum: rrnA PCL1, rrnA P1, rrnA P2b, rrnA P3

-M. phlei: rrnA PCL1, rrnA P1, rrnA P3

- Mycobacteriophage I3: pKGR25,

pKGR9, pKGR38, ORF1, ORF2, pKGR1

-Mycobacteriophage L5: $71 \mathrm{P} 2,71 \mathrm{P}_{\text {left }}$

-M. avium: avi-3, pLR7

-M. neoaurum: rrnA PCL1, rnA P1, rrnA P3

-M. absessus rrnA P1

$\bullet$ M. chelonae rrnA P1

High
-M. tuberculosis: T101, T150, rrnA PCL1, 16S rRNA, rpSL

$\bullet$ M. bovis BCG: rRNA, $18 \mathrm{~K}$, rpsL, mpb70, alpha

-M. leprae: $18 \mathrm{Kda}(\mathrm{sp}=17)$,

$18 \mathrm{Kda}(\mathrm{sp}=18)$, rpsL

$\bullet$ M. smegmatis: S6, S14, S16, S19, S30,

S35, rrn B

$\bullet$ M. fortuitum rrnA P2a

-Mycobacteriophage L5 71 P1

$\bullet$ M. neoaurum rrnA P2

-M. abscessus: rrnA P4, rrnA PCL1, rrnA P2, rrnA P3
Santis et al. [33] $]^{\mathrm{b}}$

-M. tuberculosis: T3, T26, T119, recA $\operatorname{rrnA~P1}$, KatG $\mathrm{P}_{\mathrm{A}}\left(\mathrm{sp}^{\mathrm{c}}=19\right)$, KatG $\mathrm{P}_{\mathrm{A}}(\mathrm{sp}=15)$, KatG $P_{C}(s p=22)$, KatG $P_{C}(s p=14)$, purC

$\bullet$ M. bovis BCG hsp60 P2

-M. leprae: gro E1, 36K, SOD

-M. smegmatis: alrA, S33, S69, S119,

gyr B, ask, rrnA P2

-M. paratuberculosis: pAJB303, pAJB300, pAJB73, pAJB301

-Mycobacteriophage I3 ORF1

$\bullet$ M. avium pLR7

$\bullet$ M. tuberculosis: T80, T101, T125, T129, T130, gyrA, cpn60, gyrB P3, 85A $\left(\mathrm{sp}^{\bullet}=17\right)$, 85A ( $\mathrm{sp}=22)$, gyrB P2, rrnA PCL1, 16S rRNA, glnA $(\mathrm{sp}=18), \operatorname{gln} \mathrm{A}(\mathrm{sp}=10)$, KatG $\mathrm{P}_{\mathrm{B}}$ $(\mathrm{sp}=20)$, KatG $\mathrm{P}_{\mathrm{B}}(\mathrm{sp}=22)$, purL, groE $(\mathrm{sp}=19)$, groE $(\mathrm{sp}=11)$, ahpC, $32 \mathrm{Kda}, 10 \mathrm{Kda}(\mathrm{sp}=17)$, $10 \mathrm{KDa}(\mathrm{sp}=15), 10 \mathrm{Kda}(\mathrm{sp}=8)$,

$65 \mathrm{Kda}, \mathrm{mpt64}$, rpsL, $38 \mathrm{Kda}, \mathrm{ppgK}$ $\bullet$ M. bovis BCG: rRNA, ahpC, $23 \mathrm{~K}$, mpb 64, $18 \mathrm{~K}$, rpsL, mpb70, alpha -M. leprae: $16 \mathrm{~S}$ rRNA, $18 \mathrm{Kda}(\mathrm{sp}=17)$, $18 \mathrm{kda}(\mathrm{sp}=18), 28 \mathrm{Kda}$, rpsL

$\bullet$ M. smegmatis: S4, S5, S16, S18, S19, S21, S65, recA, acetamidase, rrnA P1, RrnA P3, rpsL $(\mathrm{sp}=18)$, rpsL $(\mathrm{sp}=17)$, ahpC -M. paratuberculosis: pAJB86, pAJB125, PAJB305, pAJB304, $\mathrm{P}_{\text {AN }}$

-M. fortuitum: repA, rrnA PCL1, rnA P1, RrnA P2a, rrnA P2b, rrnA P3

-M. phlei: rrnA PCL1, rrnA P1, rnA P3

-Mycobacteriophage I3: pKGR25,

PKGR9, pKGR38, pKGR1

-Mycobacteriophage L5: $71 \mathrm{P} 2,71 \mathrm{P}_{\text {left }}, 71 \mathrm{P} 1$

-M. avium avi-3

-M. neoaurum: rrnA PCL1, rnA P1, RrnA P3, rrnA P2

- M. absessus: rrnA P1, rrnA PCL1

$\bullet$ M. chelonae: rrnA P1, rrnA PCL1

-M. tuberculosis: T6, metA

-M. bovis BCG 64K

-M. leprae $65 \mathrm{KD}$

-M. smegmatis: S6, S12, S14, S30, S35,

rrn B, rrnA PCL1

-M. fortuitum rrnA P2a

-M. phlei rrnA P2

- Mycobacteriophage I3 ORF2

- Mycobacteriophage L5 71 P1 


\begin{tabular}{lll}
\hline & Shipgelman et al. [36 $]^{\mathrm{a}}$ & Santis et al. [33] $^{\mathrm{b}}$ \\
\hline & $\bullet$ M. chelonae: $\operatorname{rnA} \mathrm{P} 2$, rrnA PCL1, \\
& rnA P3, rrnA P4 & \\
Very high & $\bullet$ M. tuberculosis gyrB P1 & $\bullet$ M. tuberculosis: T150, gyrB P1 \\
& $\bullet$ M. leprae $65 \mathrm{KD}$ & $\bullet$ M. smegmatis $\mathrm{S} 12$ \\
& $\bullet$ M. smegmatis S12 & $\bullet$ M. abscessus: rrnA P4, rrnA P2, rrnA P3 \\
& $\bullet$ M. phlei rrnA P2 & $\bullet$ M. chelonae: rrnA P2, rrnA P3, rrnA P4 \\
\hline
\end{tabular}

\footnotetext{
${ }^{\text {a }}$ Curvature maxima lying in the range [0.0-0.2], [0.2,0.4], [0.4,0.6]; and [0.6 and above] DNA curvature units are referred to as low, medium, high and very high curvature maps, respectively.

${ }^{\mathrm{b}}$ Curvature maxima lying in the range [0-5], [5-10], [10-15]; and [15 and above] units are referred to as low, medium, high and very high curvature profiles, respectively.

${ }^{\mathrm{c}}$ sp denotes spacer length in bp.
}

degrees) for the 16 different dinucleotide steps in DNA are taken from Anselmi et al. [31]. In our analysis, we have used integration step value as 31 (approx. three turns of B-DNA) in order to minimize the signal-to-noise ratio. The results of this study are also listed in Table 1 for each mycobacterial promoter. Various sub-groups of mycobacterial promoters are analyzed for the nature of curvature profile and these results are presented in Table 2. Curvature dispersion $\sigma^{2}$ quantifies the central dispersion of the local helical axes with respect to the average direction of the double helix. The $\sigma^{2}$ plot of cyclically permuted DNA sequence allows an easy alternative to experimental permutation assay for DNA tracts up to $700 \mathrm{bp}$ long. Hence, $\sigma^{2}$ plots of cyclically permuted mycobacterial promoters are prepared to see exact position of molecular bend locus. For simplicity of analysis mycobacterial promoter region is divided into the following five sub-regions: (i) region above -35 box; (ii) -35 region; (iii) spacer region; (iv) -10 region; and (v) region below -10 box. The position of molecular bend locus, for each mycobacterial promoter, with respect to the subregions specified above, is mentioned in Table 3.

3. Calladine-Dickerson Rule [34,35]: Calladine proposed four rules to understand the sequencedependent departures from classical B-DNA due to simple steric hindrance of nearest neighbor purines on opposite strands. He suggested that

Table 2

Percentage of low, medium and high curvature profiles for various sub-groups of mycobacterial promoters using: (i) experimentally determined wedge angles [36]; and (ii) energy minimized values of roll and tilt angles [33]

\begin{tabular}{|c|c|c|c|c|c|c|}
\hline \multirow[t]{2}{*}{ Mycobacterial promoters } & \multicolumn{2}{|l|}{ Low } & \multicolumn{2}{|l|}{ Medium } & \multicolumn{2}{|l|}{ High } \\
\hline & $\begin{array}{l}\text { Shipgelman } \\
\text { et al. [36] }\end{array}$ & $\begin{array}{l}\text { De Santis } \\
\text { et al. [33] }\end{array}$ & $\begin{array}{l}\text { Shipgelman } \\
\text { et al. [36] }\end{array}$ & $\begin{array}{l}\text { De Santis } \\
\text { et al. [33] }\end{array}$ & $\begin{array}{l}\text { Shipgelman } \\
\text { et al. [36] }\end{array}$ & $\begin{array}{l}\text { De Santis } \\
\text { et al. [33] }\end{array}$ \\
\hline Class I: $E$. coli $\sigma^{70}$ type (sample size $\left.=69\right)$ & 15 & 19 & 60 & 67 & 25 & 14 \\
\hline Class II: non- $E$. coli $\sigma^{70}$ type (sample size $\left.=36\right)$ & 22 & 27 & 56 & 54 & 22 & 19 \\
\hline Class II: extended -10 type $($ sample size $=24)$ & 17 & 4 & 25 & 58 & 58 & 38 \\
\hline $\begin{array}{l}\text { Having optimum }(17 \pm 1 \text { bp }) \text { spacer length } \\
\quad(\text { sample size }=79)\end{array}$ & 9 & 11 & 61 & 72 & 30 & 17 \\
\hline With high $(\geq 50 \%)$ AT content (sample size $=26)$ & 12 & 15 & 54 & 58 & 35 & 27 \\
\hline $\begin{array}{l}\text { Having } \mathrm{A}_{n} \mathrm{~T}_{m}(n+m \geq 3) \text { tract repeated in phase } \\
\text { with each other and present at the upstream of } \\
-35 \text { box }(\text { sample size }=12)\end{array}$ & 17 & 17 & 25 & 33 & 58 & 50 \\
\hline M. tuberculosis $($ sample size $=44)$ & 25 & 23 & 61 & 68 & 14 & 9 \\
\hline M. smegmatis $($ sample size $=28)$ & 21 & 25 & 50 & 50 & 29 & 25 \\
\hline Entire compilation ( sample size $=135)$ & 17 & 20 & 57 & 64 & 26 & 16 \\
\hline
\end{tabular}


Table 3

Location of molecular bend locus with reference to the following sub-regions in the mycobacterial promoter (such as MT, $M$. tuberculosis; MB, M. Bovis BCG; ML, M. leprae; MS, M. smegmatis; MP, M. paratuberculosis; MF, M. fortuitum; MH, M. phlei; MI, Mycobacteriophage I3; MY, Mycobacteriophage L5; MV, M. avium; MN, M. neoaurum; MA, M. abscessus; MC, M. chelonae) sequence: (i) region above -35 box; (ii) -35 region; (iii) spacer region; (iv) -10 region; and (v) region below -10 box

\begin{tabular}{|c|c|c|c|c|}
\hline Region above -35 box & -35 region & Spacer region & -10 region & Region below -10 box \\
\hline \multicolumn{5}{|c|}{ Promoters whose transcription start site is determined } \\
\hline MT T180 & MT T119 & MT T130 & MT T101 & MT T3 \\
\hline MT recA & MT T125 & MT cpn60 & MS S14 & MT T6 \\
\hline MT $85 \mathrm{~A}\left(\mathrm{sp}^{\mathrm{a}}=17\right)$ & MT T129 & MT gyrB P1 & MS rpsL $(\mathrm{sp}=17)$ & MT T26 \\
\hline MT KatG $P_{C}(s p=22)$ & MT $85 \mathrm{~A}(\mathrm{sp}=22)$ & MT gyrB P2 & MP pAJB86 & MT T150 \\
\hline MT purC & MT purL & MT katG $P_{A}(s p=19)$ & MY 71P2 & MT rrnA P1 \\
\hline ML 16S rRNA & MS S4 & MT katG $P_{A}(s p=15)$ & - & MT gyrA \\
\hline MS S69 & MS S5 & MT katG $P_{B}(s p=20)$ & - & MT gyrB P3 \\
\hline MS gyrB & MS S19 & MT katG $P_{B}(s p=22)$ & - & MT rrnA PCL1 \\
\hline MS ask & MS S21 & MB hsp60 P2 & - & MT 16S rRNA \\
\hline MS rrnA P1 & MS S119 & MS S6 & - & MT $g \ln A(s p=18)$ \\
\hline MS rrnA P2 & MS rrnB & MS S12 & - & MT $g \ln A(s p=10)$ \\
\hline MP pAJB300 & MA rrnA P4 & MS S16 & - & MT KatG $P_{C}(s p=14)$ \\
\hline MF rrnA PCL1 & $\mathrm{MC} \operatorname{rrnA} \mathrm{P} 2$ & MS S18 & - & ML $18 \mathrm{kDa}(\mathrm{sp}=17)$ \\
\hline MH rrnAPCL1 & MC $\operatorname{rrnA~P3}$ & MS S30 & - & ML $18 \mathrm{kDa}(\mathrm{sp}=18)$ \\
\hline- & - & MS S33 & - & MS alrA \\
\hline- & - & MS S35 & - & MS S65 \\
\hline- & - & MP pAJB303 & - & $\mathrm{MS}$ recA \\
\hline- & - & $\mathrm{MP} \mathrm{P}_{\mathrm{AN}}$ & - & MS acetamidase \\
\hline- & - & MF repA & - & MS rrnA P3 \\
\hline- & - & MY 71P1 & - & MS rrnA PCL1 \\
\hline- & - & MA rrnA P1 & - & MS rpsL $(\mathrm{sp}=18)$ \\
\hline- & - & MA rrnAPCL1 & - & MP pAJB125 \\
\hline- & - & MA rrnA P2 & - & MP pAJB305 \\
\hline- & - & MA rrnA P3 & - & MP pAJB304 \\
\hline- & - & MC rrnA P1 & - & MP pAJB73 \\
\hline- & - & MC rrnA PCL1 & - & MY 71P left \\
\hline- & - & - & - & MN rrnAPCL1 \\
\hline- & - & - & - & MC rrnA P4 \\
\hline $16 \%$ & $16 \%$ & $30 \%$ & $6 \%$ & $32 \%$ \\
\hline \multicolumn{5}{|l|}{ Putative promoters } \\
\hline MT $32 \mathrm{kDa}$ & MT ahpC & MT $10 \mathrm{kDa}$ & ML SOD & MT groE \\
\hline ML $28 \mathrm{kDa}$ & MT metA & MT $38 \mathrm{kDa}$ & MI pKGR25 & MT groE \\
\hline MF rrnA P1 & MT rpsL & MT ppgK & $\mathrm{MN}$ rrnA P2 & MT $10 \mathrm{kDa}$ \\
\hline MN rrnA P1 & MB ahpC & MB alpha & - & MT $10 \mathrm{kDa}$ \\
\hline- & MB rpsL & MI pKGR38 & - & MT $65 \mathrm{kDa}$ \\
\hline- & ML $65 \mathrm{kDa}$ & MI ORF2 & - & MT mpt64 \\
\hline- & ML 36K & MV pLR7 & - & MB rRNA \\
\hline- & ML rpsL & - & - & MB $23 \mathrm{~K}$ \\
\hline- & MS ahpC & - & - & MB mpb64 \\
\hline- & MH rrnA P2 & - & - & MB $18 \mathrm{~K}$ \\
\hline- & MI pKGR9 & - & - & MB $64 \mathrm{~K}$ \\
\hline- & - & - & - & $\mathrm{MB}$ mpb70 \\
\hline- & - & - & - & ML groE1 \\
\hline- & - & - & - & MP pAJB301 \\
\hline- & - & - & - & MF rrnA P2a \\
\hline- & - & - & - & MF rrnA P2b \\
\hline- & - & - & - & MF rrnA P3 \\
\hline - & - & - & - & MH rrnA P1 \\
\hline
\end{tabular}




\begin{tabular}{lllll}
\hline Region above -35 box & -35 region & Spacer region & -10 region & Region below -10 box \\
\hline- & - & - & - & MH rrnA P3 \\
- & - & - & - & MI ORF1 \\
- & - & - & - & MI pKGR1 \\
- & - & - & - & MV Avi-3 \\
- & - & - & - & MN rrnA P3 \\
$8 \%$ & $23 \%$ & $15 \%$ & $6 \%$ & $48 \%$ \\
\hline
\end{tabular}

${ }^{\mathrm{a}} \mathrm{sp}$ denotes spacer length in bp.

the DNA chains may overcome these steric clashes in four possible ways: (i) the helix twist angle may be reduced; (ii) the base pairs can rotate along their long axes; (iii) the DNA backbone can shift sideways towards the pyrimidines; and (iv) the propeller twist can be suppressed. Dickerson quantified this by constructing four sum functions $\left(\Sigma_{1}-\Sigma_{4}\right)$, by means of which the base sequence can be used to calculate the expected local variation in helix twist $\left(\Sigma_{1}\right)$, base plane roll $\left(\Sigma_{2}\right)$, torsion angle difference at the two ends of the base pair $\left(\Sigma_{3}\right)$, and flattening of propeller twist $\left(\Sigma_{4}\right)$. DNA helical structure variation at the molecular bend locus is studied here for mycobacterial promoters using Calladine-Dickerson rules. For this analysis, we have taken 11-bp-long sequence stretch obtained by taking five nucleotides on either side of the molecular bend locus of each mycobacterial promoter. For brevity, only $\Sigma_{1}$ function plots for the promoters whose TSS is mapped are shown in Fig. 1.

4. Propeller twist [30]: it is known that different types of dinucleotide step have different levels of conformational flexibility, which is very closely related to the Propeller-twist. Propeller twist values are obtained from X-ray crystallography of DNA oligomers. Dinucleotides with a large propeller-twist have a tendency to be more rigid than dinucleotides with low propeller twist. Higher (less negative) values correspond to higher flexibility. Flexibility profile was plotted using the propeller twist values from X-ray crystallography of DNA oligomers for overlapping dinucleotides.

5. DNase I derived bendability parameters [29]: the productive binding of bovine pancreatic deoxyribonuclease I (DNase I) requires DNA to be bent toward the major groove (positive roll). Base sequences that are flexible or inherently bent towards the major groove should therefore be more accessible to DNase I cleavage. DNase I cutting frequencies on naked DNA can be used as a quantitative measure of anisotropic bendability (major groove compressibility). Bendability profile was calculated using DNase I derived bendability parameters for overlapping trinucleotides of each mycobacterial promoter sequence.

6. Location preference [23]: from experimental investigations of the positioning of DNA in nucleosomes, it has been found that certain trinucleotides have strong preference for having minor grooves facing either towards or away from the nucleosome core. Based on the premise that flexible sequences can occupy any rotational position on nucleosomal DNA, while rigid sequences will be restricted in rotational location. We have calculated DNA flexibility profile using these location preference values for mycobacterial promoters at each position considering overlapping trinucleotides.

\section{Results and discussion}

The curvature distribution for various mycobacterial promoters as calculated using different models show similar trends. In order to aid the analysis the results obtained using: (i) experimentally determined wedge angles; and (ii) energy minimized values of roll and tilt angles, have been compared. The extent of curvature obtained using these models has been classified in terms of low, medium or high curvature and the results of the two models corroborate each other for most of the promoters barring a few promoter entries (e.g. M. tubercu- 
Region 1:

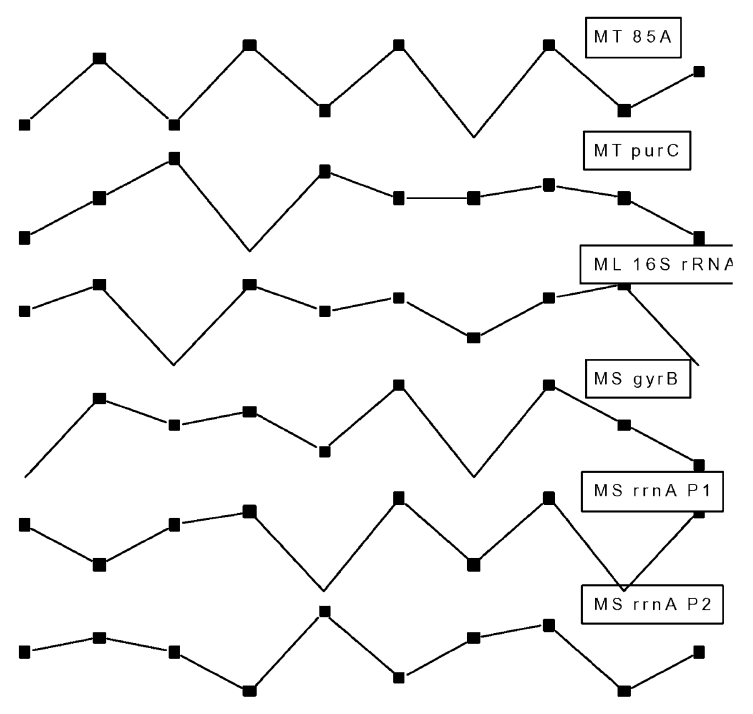

Region 2:

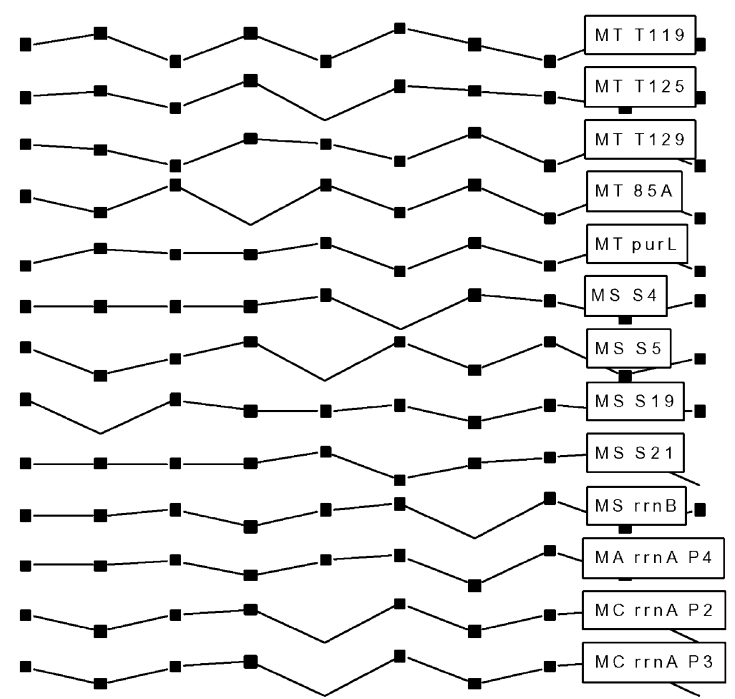

Region 3:
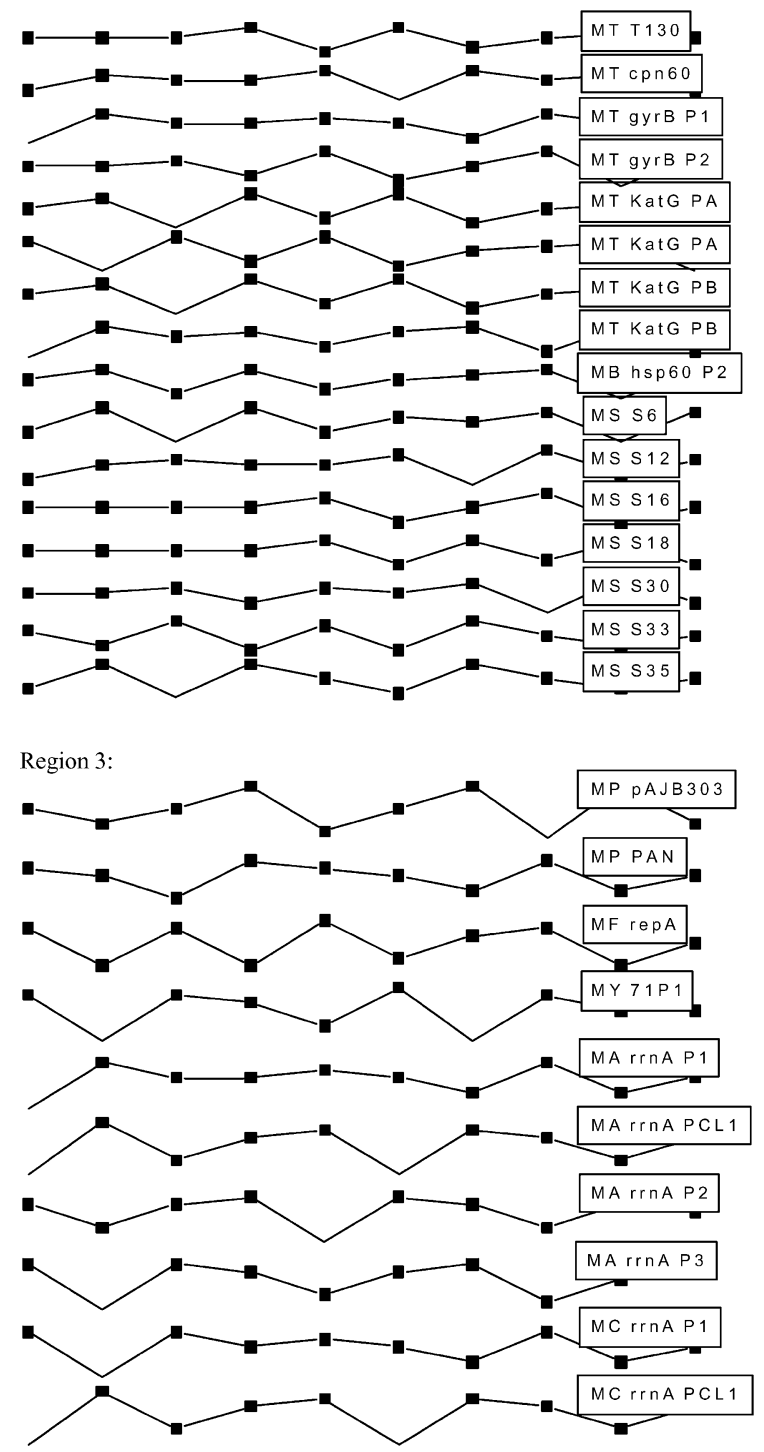

Fig. 1. $\Sigma_{1}$ function plots for the true mycobacterial promoters, sub-grouped depending upon the location of the molecular bend locus.

losis T3, T6, T101, T129, T130, recA, rrnA P1, gyrA, cpn60, rrnA PCL1, 16S rRNA, metA, rpsL, etc.) where the prediction of the two models differ.

In order to obtain a better insight for the results obtained by these two models, mycobacterial pro- moters are sub-divided into various groups. These groups are as follows: (i) Class I, mycobacterial promoters resembling to $E$. coli $\sigma^{70}$ type promoters; (ii) Class II, mycobacterial promoters which are different from E. coli $\sigma^{70}$ type promoters, and 
Region 4:

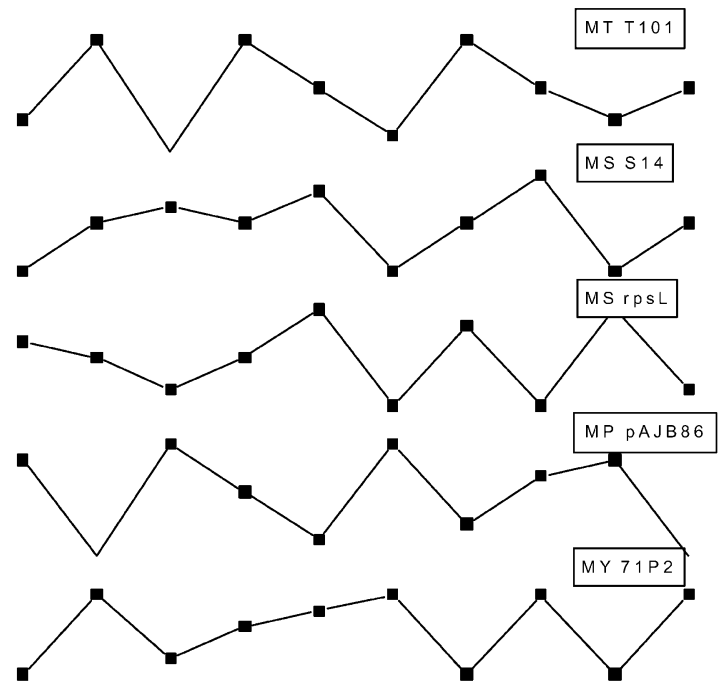

Region 5:

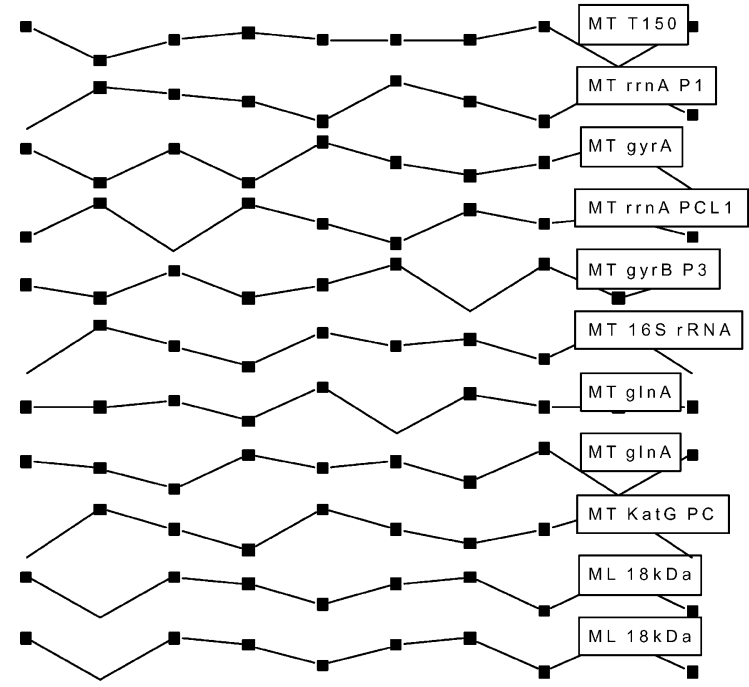

Region 5:

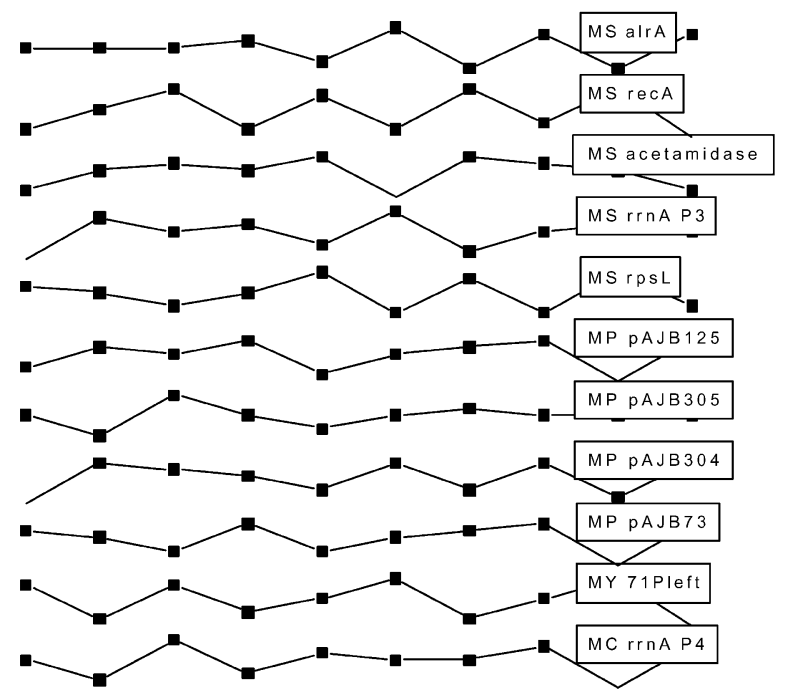

Fig. 1 (Continued.

constituting a class known as typical mycobacterial promoters; (iii) Class III, extended -10 promoters; (iv) mycobacterial promoters having optimum (17 $\pm 1 \mathrm{bp)}$ spacer length; (v) mycobacterial promoters having high $(\geq 50 \%)$ AT content; (vi) mycobacterial promoters having $\mathrm{A}_{n} \mathrm{~T}_{m}(n+m \geq 3)$ tract repeated in phase with each other and present at the upstream of the -35 box; (vii) M. tuberculosis promoters; (viii) M. smegmatis promoters; and (ix) entire mycobacterial promoter compilation. The curvature analysis of promoters classified in these groups are listed in Table 2. From Table 2, it can be seen that $E$. coli $\sigma^{70}$ type mycobacterial promoters have $15 \%(19 \%), 60 \%(67 \%)$ and $25 \%$ 
(14\%) of low, medium, and high curvature profiles using curvature models of Shpigelman et al. [36] and De Santis et al. [33]. This distribution indicates that mycobacterial promoters resembling to E. coli $\sigma^{70}$ type (Class I) have nearly $81 \% \quad(85 \%)$ sequences having medium and high curvature profiles. Very few, i.e. 19\% (15\%) promoter sequences are having low curvature profiles. Considering percent distribution of curvature existing among E. coli $\sigma^{70}$ type mycobacterial promoters, we can say that these promoters might be having good promoter activity. The analysis also indicates that the Non-E. coli $\sigma^{70}$ type mycobacterial (Class II) promoters have $22 \%(27 \%), 56 \%(54 \%)$ and $22 \%$ (19\%) of low, medium, and high curvature profiles (using both curvature models). This group of mycobacterial promoters has comparatively higher percent of low curvature profiles indicating that Non-E. coli $\sigma^{70}$ type mycobacterial promoters might be poorly expressed compared to $E$. coli $\sigma^{70}$ type mycobacterial promoters. The curvature models applied to the extended -10 (Class III) promoters show $17 \%(4 \%), 25 \%(58 \%)$ and $58 \%$ (38\%) of low, medium, and high curvature profiles. The percent distribution of these promoters indicates that very few of these promoters have low curvature profiles. Extended -10 promoters might therefore have reasonably high promoter activity. M. tuberculosis T101, M. smegmatis S6, $\mathrm{S} 16$, and S19 promoters are extended -10 promoters, which are strongly curved. For such mycobacterial promoters sequence of the -35 region seems to be less important due to the presence of an extended TG motif in the immediate neighborhood of the -10 box along with the high curvature existing within it. Mycobacterial promoters lacking a consensus sequence at -35 and are curved are M. tuberculosis T150, M. smegmatis S12, S14, S30 and S35. Here, the curvature along with the -10 region might be useful for promoter activity although they do not possess a TG motif in the immediate neighborhood of the -10 box. The mycobacterial promoters having optimum $(17 \pm 1$ bp) spacer length have $9 \%(11 \%)$ of sequences having low curvature profiles by both the models. The majority of sequences from this class has a curved structure. The favorable flexibility and/or curvature of DNA may compensate somewhat for a sub-optimal spacing of 16 or 18 bp between 35 and -10 regions during transcription initiation. The mycobacterial promoters with high percentage of AT have $12 \%(15 \%), 54 \%(58 \%)$ and $35 \%$ $(27 \%)$ of sequences possess low, medium and high curvature profiles, respectively. The occurrence of curvature is obvious for the majority of sequences from this class due to their high percentage of AT content. Among mycobacterial promoters with $\mathrm{A}_{n} \mathrm{~T}_{m}(n+m \geq 3)$ tract repeated in phase with each other and present at the upstream of the -35 box, $58 \%(50 \%)$ of sequences have high curvature trends. These promoters having upstream sequences, which can be expected to produce curvature in the DNA helical axis might be transcriptionally active promoters. M. tuberculosis promoters have $14 \%$ (9\%), and M. smegmatis promoters have $29 \%$ $(25 \%)$ of high curvature profiles. Such percentage distribution may be one of the causative factors for M. smegmatis to express better than M. tuberculosis. For the analysis performed in Table 2, it is important to realize that the percentage value of curvature predictions by both models sometimes differ significantly due to different conditions defined for low, medium, and high curvature profiles; and in a few cases predictions by the two models lie on the boundary conditions of low and medium, or medium and high curvature profiles. The sample size considered in this analysis is also small, and can cause large differences in the predictions by these two models. Results listed in Table 2 should only be used to see qualitative and semi-quantitative trends.

According to CURVATURE software, curvature maxima for $M$. tuberculosis gyrB $\mathrm{P} 1, M$. bovis $B C G$ alpha, $M$. fortuitum rnA P1, Mycobacteriophage L5 71P1, M. neoaurum rrnA PCL1, and rrnA P3 promoters lies above 0.3 DNA curvature units and it is present between the -35 and -10 regions. It will be interesting to study the transcription initiation mechanism in these promoters because in E. coli it is shown that the curvature between -35 and -10 regions seems to correlate significantly with promoter activity. In such cases the curved structure of promoter DNA enhances the binding of $E$. coli RNA polymerase to the promoter, when the curve is oriented correctly relative to the potential -10 and -35 regions, 
and it also facilitates unwinding of the -10 region by thermal motion, as the DNA vibrates back and forth in solution between the twisted and curved forms [11].

$\sigma^{2}$ plots of cyclically permuted mycobacterial promoters should allow an alternative to the experimental permutation assay for determining molecular bend locus of a mycobacterial promoter sequence. The model has been successful in predicting the experimental results for other systems $[33,38,39]$, while promoters analyzed here have not been subjected to any such experimental investigations and hence, the theoretical predictions could not be tested. In Table 3, we have evaluated the percent occurrence of position of molecular bend locus in the (i) region above -35 box, (ii) -35 region, (iii) spacer region, (iv) -10 region, and (v) region below the -10 box. For this analysis, we have separated the entire promoter compilation into two groups: (i) promoters whose TSS is mapped (true promoters); and (ii) putative promoters. According to the percentage distribution for true promoters, the molecular bend locus lies predominantly in the spacer region and the region below the -10 box. Sixteen, 16, 30, 6 and $32 \%$ of true mycobacterial promoter sequences show that their molecular bend locus lies in the region above the -35 box, -35 region, spacer region, -10 region, and the region below the 10 box, respectively. For putative promoters, 8, 23, 15,6 and $48 \%$ of sequences show their molecular bend locus in a region above the -35 box, -35 region, spacer region, -10 region, and a region below the -10 box, respectively. Thus, for true as well as putative mycobacterial promoters, the spacer region and region below the -10 box seems to be of frequent occurrence for the location of molecular bend locus. Similar studies by Nair and Kulkarni [40] on E. coli promoter sequences showed that $60 \%$ of these promoters have their minima (molecular bend locus) lying in the spacer region. However, for mycobacterial promoters, the position of the molecular bend locus can occur with varying percent distribution at a region above the -35 box, -35 region, spacer region and a region below the -10 box. Thus, mycobacterial promoters show variation in the position of the molecular bend locus compared to E. coli promoters.

The Calladine and Dickerson rule $\left(\Sigma_{1}-\Sigma_{4}\right)$ gives a way of revealing possible structural homology between regions of DNA, when the similarity is not obvious by direct comparison of sequence alone. The helical structure variation at the molecular bend locus for the true mycobacterial promoters is sub-grouped according to the position of molecular bend locus. Thus, Fig. 1 is subdivided into five plots. The helical structure variation obtained using the $\Sigma_{1}$ function at the molecular bend locus lying in the (i) region above the -35 box, (ii) -35 region, (iii) spacer region, (iv) 10 box, and ( $\mathrm{v}$ ) region below the -10 box shows that each sub-group has structural similarity within that particular sub-group. The other sum functions also uphold the structural similarities (results not shown). The analysis of the sequence at the minima reveals that there exists homology among these sequences irrespective of the exact position of minima. The regions that are localized for mycobacterial promoters show significant commonality in structure, which is evident from the $\Sigma_{1}$ function plot. There seems to exist some structural commonalties among the each sub-group of mycobacterial promoters. We can therefore group the promoters based on the common structural features and advocate the notion of 'consensus structure' suggesting their common biological significance. The variation from these consensus structures can account for varying strength of the promoters. Such an analysis might help us in designing experiments to define the exact location and function of a promoter.

Although the entire mycobacterial promoter compilation has been analyzed using other curvature models $[23,29,30]$, the results obtained using only three models are presented.

Mycobacterial promoters that are strongly curved are $M$. tuberculosis T150, and gyrB P1; $M$. Leprae 65KD; M. smegmatis S6, S12, S14, S30, $\mathrm{S} 35$, and $\mathrm{rrnB} ; M$. Phlei rrnA P2; M. abscessus rrnA P4, rrnA P2, and rrnA P3; M. chelonae rrnA $\mathrm{P} 2$, rrnA P3, and $\operatorname{rrnA}$ P4. Fig. 2 shows the curvature map expressed in DNA curvature units of these promoters using CURVATURE software. The curvature maxima of these curvature maps 

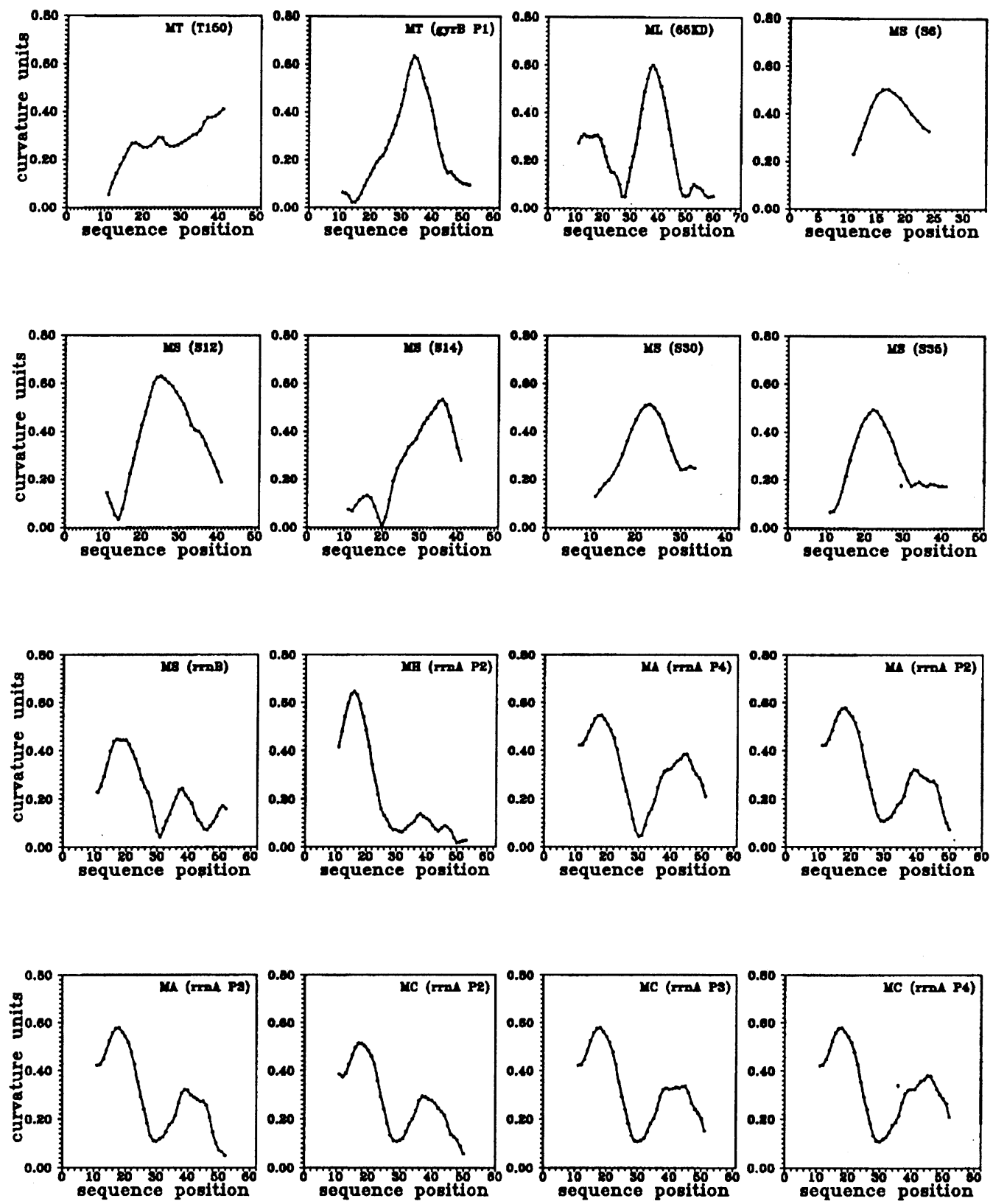

Fig. 2. Curvature map obtained using experimentally determined wedge angles for mycobacterial promoters. Curvature is expressed in DNA curvature units [37] where one curvature unit corresponds to the mean DNA curvature in the crystalline nucleosome (1/42.8 $\AA$ ).

correspond to region having more curved structure. Fig. 3a,b presents the curvature analysis using energy-minimized values of roll and tilt angles.
The curvature vector is a complex function of the sequence with the modulus representing the deviation and the phase indicating the relative direc- 

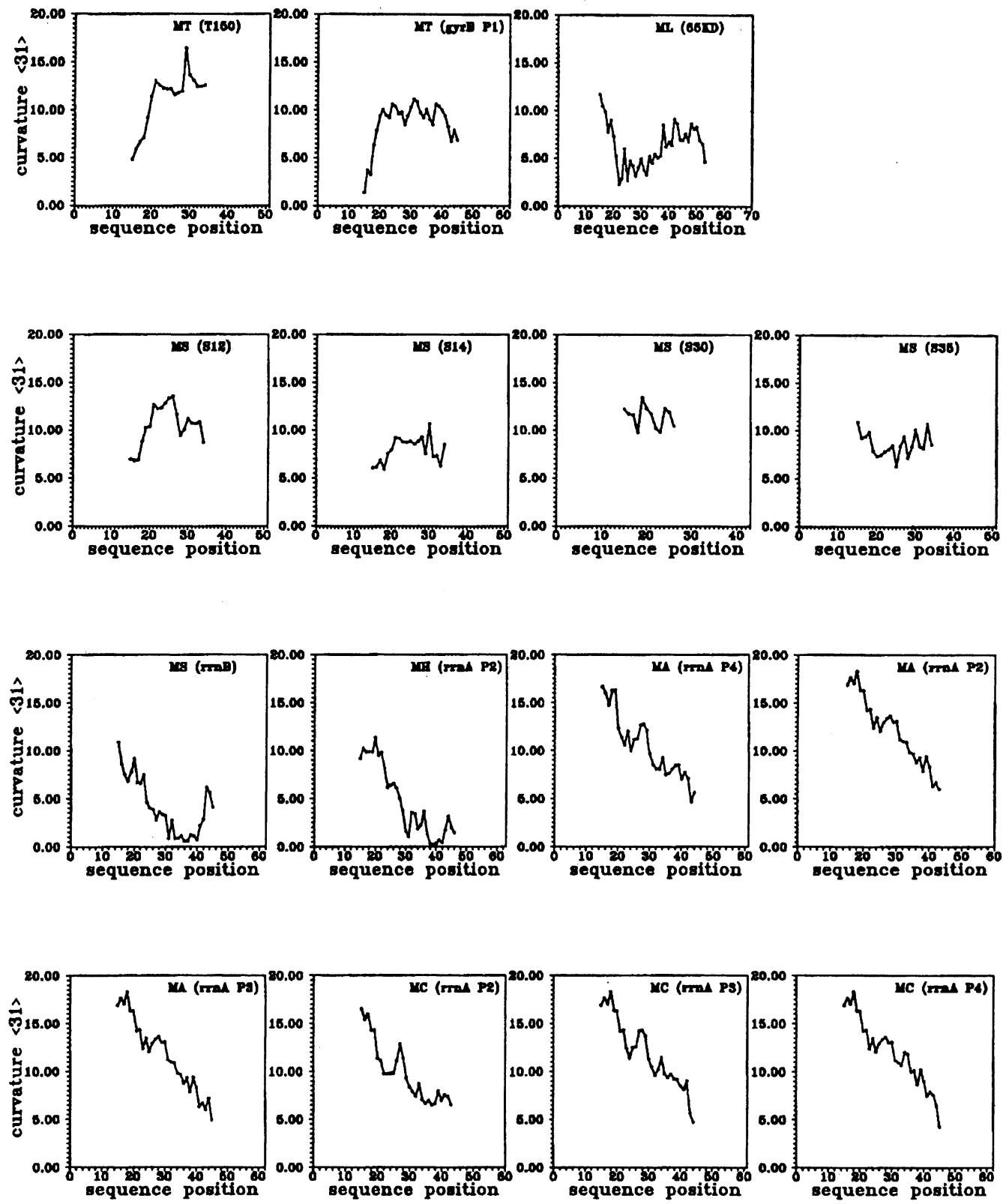

Fig. 3. (a) Curvature profiles obtained using energy minimized values of roll and tilt angles for mycobacterial promoters. The curvature is reported as $|C|$, the curvature modulus averaged over $31 \mathrm{bp}$ (M. smegmatis S6 is excluded from this plot as grid size used for it is $21 \mathrm{bp}$ ). (b) Relative phase profiles of the mycobacterial promoters.

tion. The curvature diagrams for these mycobacterial promoters clearly show a DNA tract characterized by both a high curvature modulus (see Fig. 3a) and a constant phase (Fig. 3b). Fig.
4 shows flexibility profiles based on propeller twist values from X-ray crystallography of DNA oligomers. Dinucleotides with a large propellertwist have a tendency to be more rigid than 

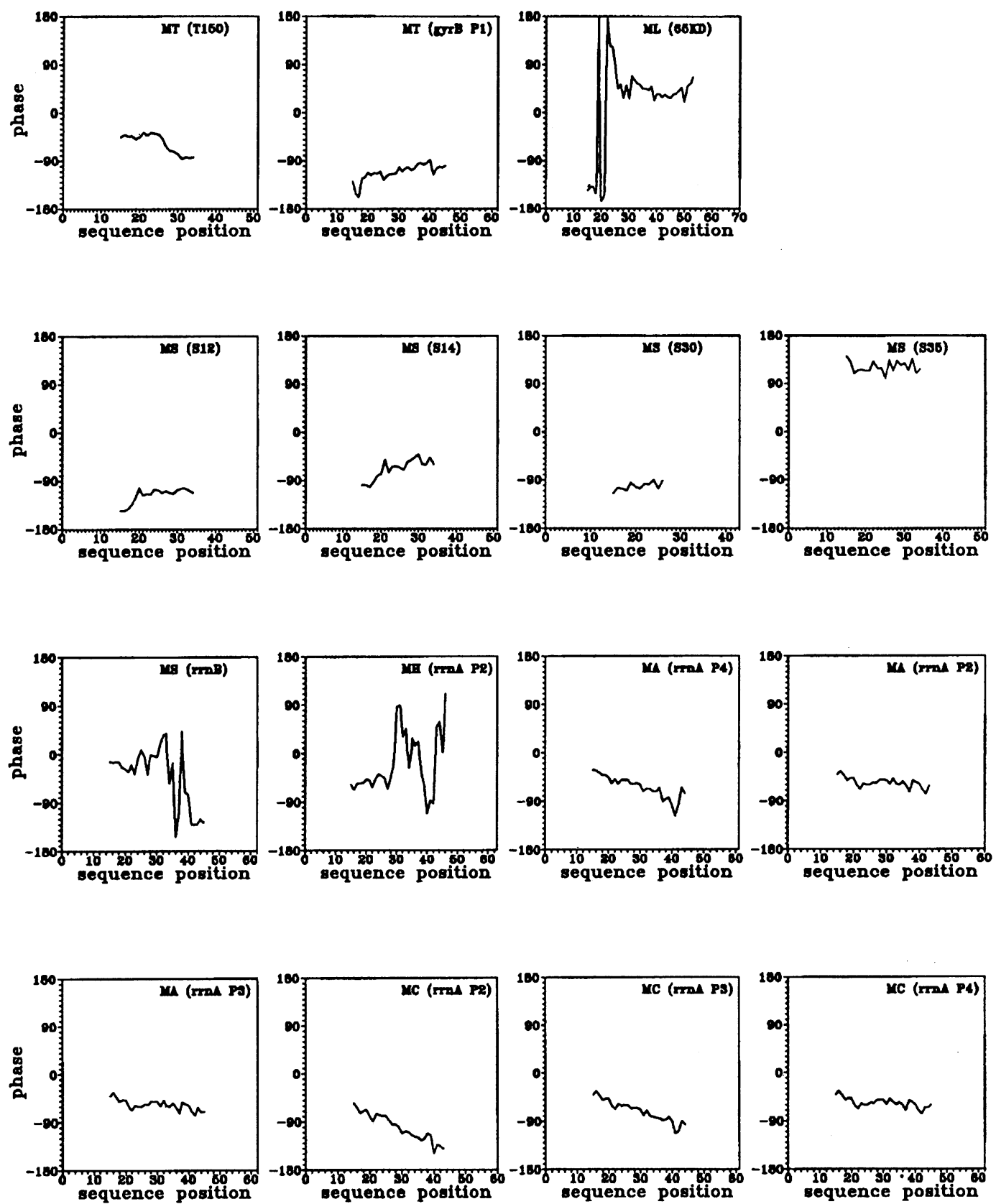

Fig. 3 (Continued).

dinucleotides with low propeller-twist. Thus, sequence positions corresponding to higher (less negative) values represent regions of higher flexibility for mycobacterial promoter. Fig. 5 presents flexibility profile calculated using a trinucleotide model based on preferred sequence location on nucleosomes. Sequence positions corresponding to lower values of location preference represent a 

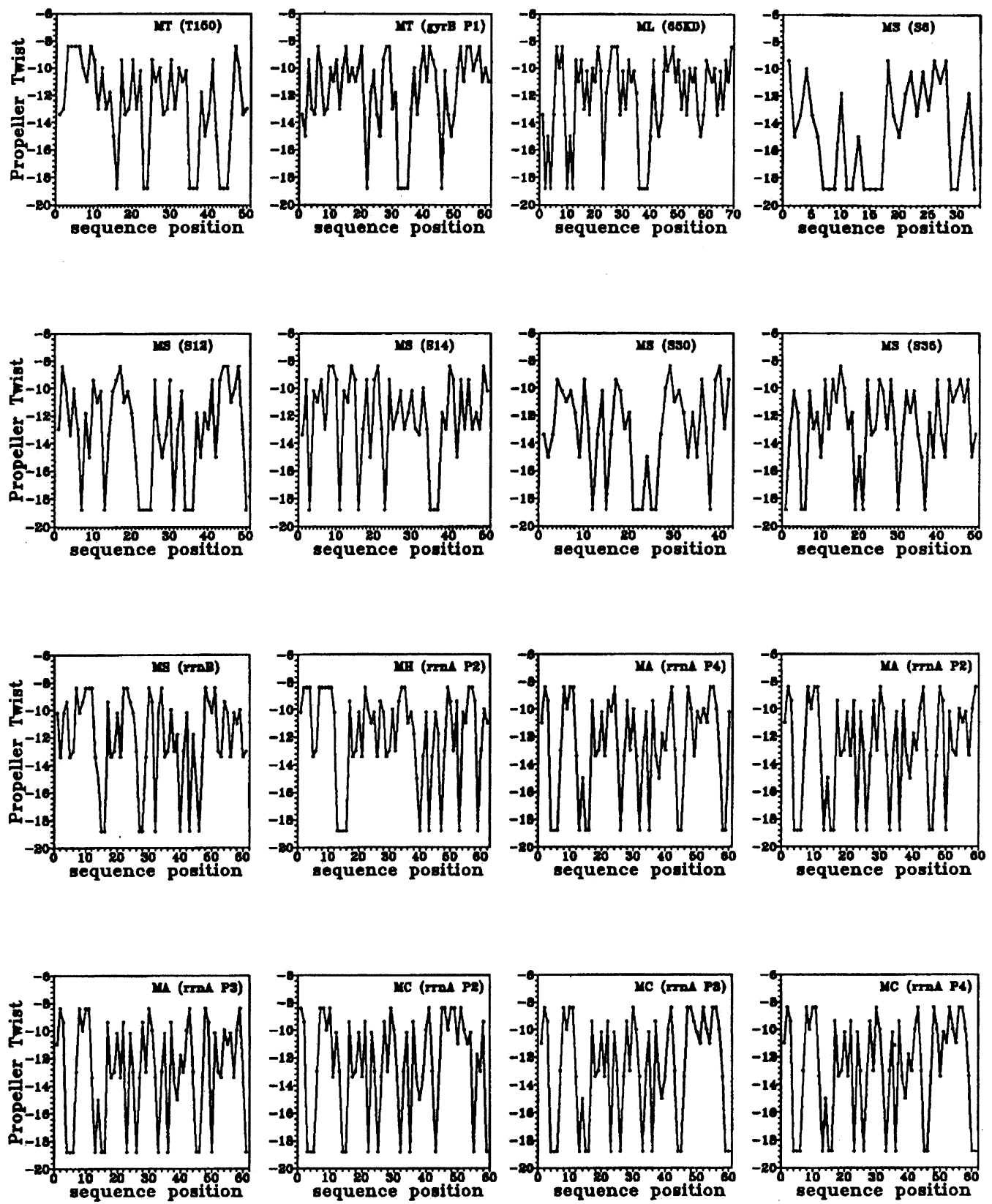

Fig. 4. Flexibility profile calculated using propeller-twist values obtained from X-ray crystallography of DNA oligomers for mycobacterial promoters.

more flexible region of the mycobacterial promoter, which have less preference for being positioned specifically. Fig. 6 shows a bendability profile in mycobacterial promoters calculated using DNase I derived bendability parameters. A sequence position corresponding to higher bendability parame- 

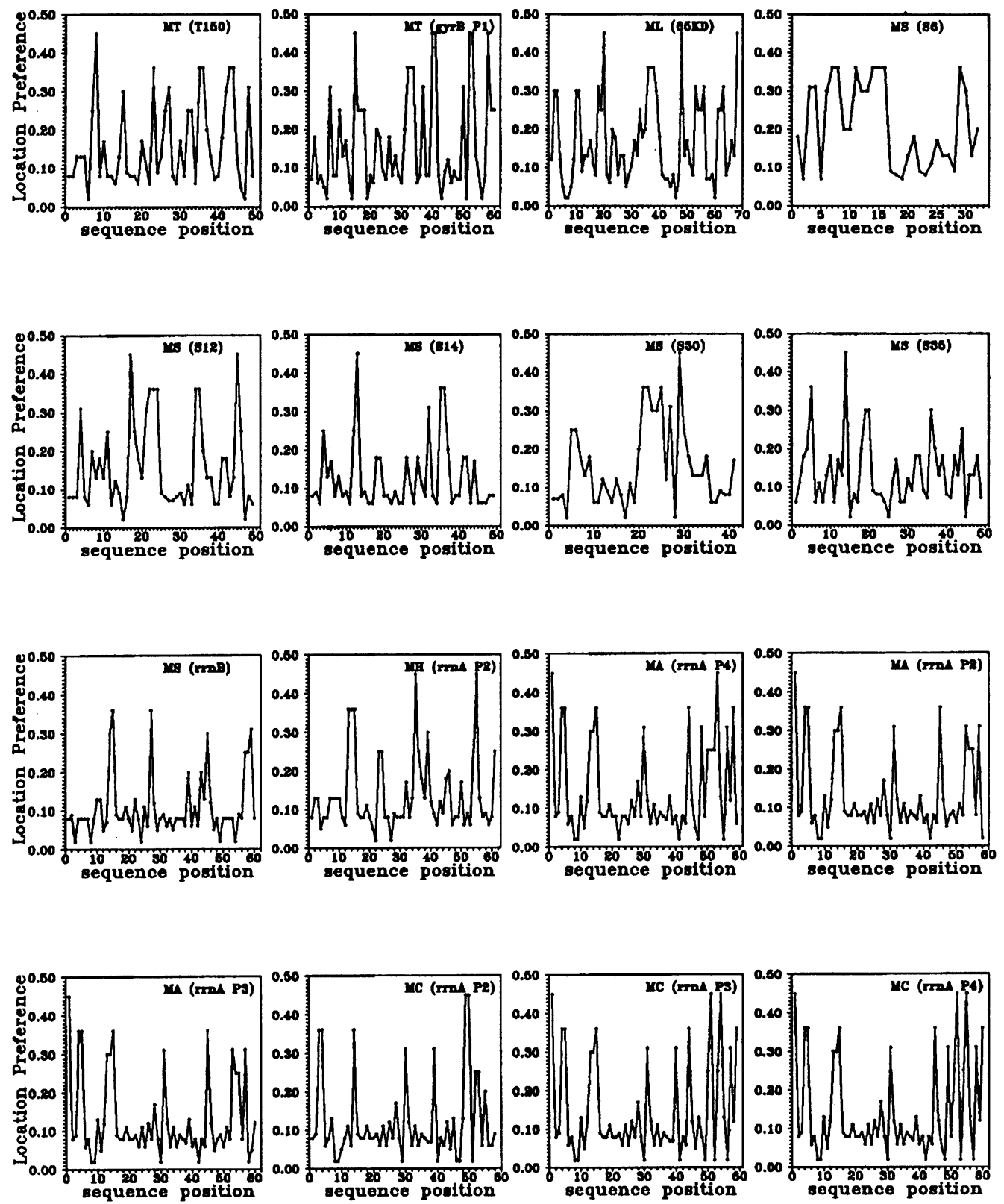

Fig. 5. Flexibility profile calculated using trinucleotide model based on preferred sequence location on nucleosomes for mycobacterial promoters.

ters represent higher propensity for major groove compressibility of the mycobacterial promoter. Essentially, all the models predict similar behavior for these promoters. Thus, a nucleotide sequence position corresponding to high (low) curvature shows a high (low) curvature trend with all the other models. Mycobacterial promoters of $M$. abscessus $\operatorname{rrnA} \mathrm{P} 4, \operatorname{rrnA} \mathrm{P} 2$, and $\operatorname{rrnA} \mathrm{P} 3 ; M$. 

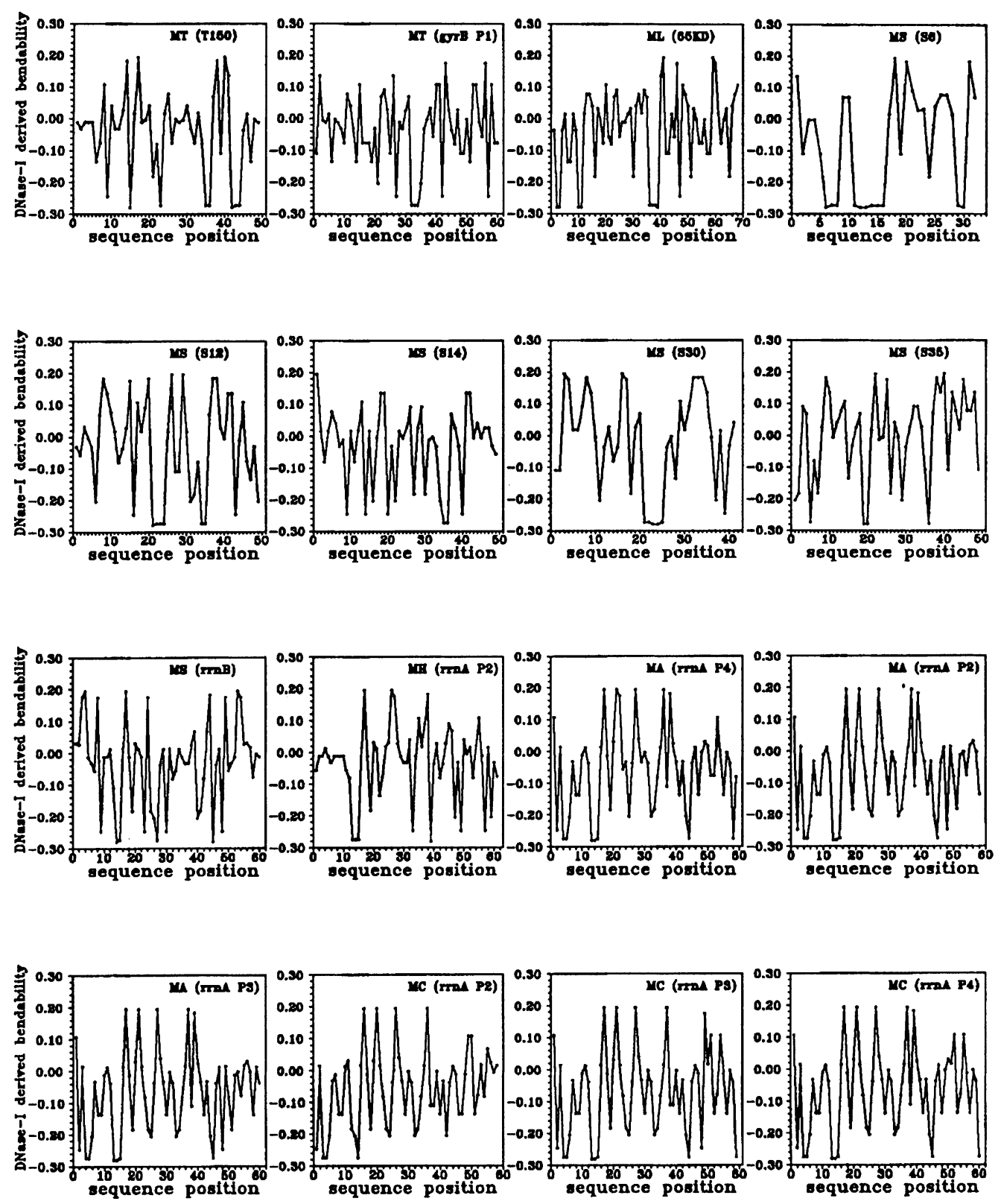

Fig. 6. Bendability profile calculated using DNase I derived bendability parameters for the mycobacterial promoters.

chelonae $\operatorname{rrnA} \mathrm{P} 2, \operatorname{rrnA} \mathrm{P} 3$, and rnA $\mathrm{P} 4$ have similar curvature trends as their nucleotide sequence shows maximum homology with each other. The similar curvature trends suggest a common mechanism for transcription initiation.
Regions with high DNA curvature would be expected to exhibit anomalous mobility by the gel electrophoresis assay. It will be of interest to examine fragments containing these regions for the structural feature of DNA curvature, and the 
corresponding functional feature of transcriptional activation. Plasmids containing stiff, flexible or curved DNA structure near the cleavage site of commonly used restriction enzymes can be helpful for studying the role of DNA structure in transcription mechanism of mycobacterial promoters.

Thus, analysis of DNA curvature distribution for mycobacterial promoters reveals the following important features. (i) The curvature distribution for various mycobacterial promoters calculated using different models show similar trends. (ii) Mycobacterial promoters resembling to E. coli $\sigma^{70}$ type have nearly $81 \%$ ( $85 \%$ ) sequences having medium and high curvature profiles. (iii) Non- $E$. coli $\sigma^{70}$ type mycobacterial promoters have com paratively higher percent of low curvature profiles. (iv) Very few of the extended -10 promoters have low curvature profiles. (v) Mycobacterial promoters having $\mathrm{A}_{n} \mathrm{~T}_{m}(n+m \geq 3)$ tract in the upstream region of the -35 box and repeated in phase with each other have high curvature profiles. (vi) M. smegmatis promoters have high curvature profiles compared to $M$. tuberculosis promoters.

Experimental studies based on curvature distribution and its role in transcription mechanism for particular mycobacterial promoter(s) or representative examples from various groups of mycobacterial promoters showing some distinct features, will throw light on our understanding of transcription mechanism of Mycobacteria.

\title{
Appendix A: Compilation of Mycobacterial promoters ${ }^{1}$
}

\begin{abstract}
M. tuberculosis
T3 ATCGACGGCCACGGCTGGTCTAGGACGAGGTACCCGG(TAACAT)GCTGGGC[G]

T6 CCGTCCAGTCTGGCAGGCCGGAAACATCGGTCAGCAGA(TAGGCT)TTACCA[G]

T26 CTGCGAGCATCATATGCCGCGTGCGTGGTGATGCGGCAG(GATGTT)GGACC[A]

T180 GATCACTCCGAGCATGCGCCCATTGTTGTGCATAGGG(CAGGAT)GCCCTG[G]

T101 AGCGATCGCAGCCGACGTGATACCTGACCGTTGTTGA(TAGTGT)CGGCGGC[A]

T119 CCCCGTGCTCGTAGTAGGCGTCCAGCCGACCCGCEGC(TACCAT)GCACAAG[T]

T125 CCGAGGTAAGGACTGAGCATGGGCCCGATAAAGTGAC(TATTAT)GGATTTC[T]

T129 ACTCGCGGCAGATTACGCCGACGGTTCCTGGCGTGG(TTCAAT)ATTCGCCG[A]

T130 ACTCCAACAGGTCGATAACCTCCTGCGCCTGCTCGTC(TATGCT)GCGATCC[G]

T150 GACCCCCGCCACGTATTGACACTTTGCGACACGCTTT(TATCAT)TTTCCGA[C]

RecA TTCGGAGCAGCCGAC(TTGTCA)GTGGCTGTC(TCTAGT)GTCACGGCC[A]ACCGACCGAT

RrnA $P 1$ GAGAACCTGGTGAGT(CTCGGT)GCCGAGATCGAACGGG(TATGCT)GTTAGGC[G]ACGGTCACCT

GyrA GATGGGCGAGGACGT(CGACGC)GCGGCGCAGCTTTATCA(CCCGCA)ACGCCAA[G]GATGTTCGGT

Cpn60 CCCCGGCGATCCCCG(TGCTCA)CCACGGGTGATTTCCGG(GGCGGC)ATGCGTT[A]GCGGACTAGC

GyrB P1 GATGTCCGACGCACG(GCGCGG)TTAGATGGGTAAAAACG(AGGCCA)GAAGATC[G]GCCCTGGCGC

GyrB P3 CAAGGGGCCTCGCCA(TATTGC)CGGTAGGGGTCCGCGCG(ACACCT)ACGGATA[A]CACGTCGATC

85A GAAGTTGTGGTTGAC(TACACG)AGCACTGCCGGGCCCAG(CGCCTG)CAGTCTG[A]CCTAATTCAG

85A CGCCCGAAGTTGTGG(TTGACT)ACACGAGCACTGCCGGGCCCAG(CGCCTG)CAGTCTG[A]CCTAATTCAG

GyrB $P 2$ AGCGGTTGGCAACGA(TGTGGT)GCGATCGCTAAAGATCAC(CGGGCC)GGCACC[A]TCGTGGCGCA

RrnA PCL1 TGACCGAACCTGGTC(TTGACT)CCATTGCCGGATTTGTAT(TAGACT)GGCAGG[G]TTGCCCGAAA

$16 S$ rRNA TGACCGAACCTGGTC(TTGACT)CCATTGCCGGATTTGTAT(TAGACT)GGCAGG[G]TTGCCCCGAA

GlnA TCGGCATGCCACCGG(TTACGA)TCTTGCCGACCATGGCCC(CACAAT)AGGGCCGGGG[A]GACCCGGCGT

GlnA CCACCGGTTACGATC(TTGCCG)ACCATGGCCC(CACAAT)AGGGCCGGGG[A]GACCCGGCGT

KatG $P_{A} \quad$ GGTCATCTACTGGGG(TCTATG)TCCTGATTGTTCGATATCC(GACACT)TCGCGATC[A]CATCCGTGAT

$K a t G P_{A} \quad$ ATCTACTGGGGTCTA(TGTCCT)GATTGTTCGATATCC(GACACT)TCGCGATC[A]CATCCGTGAT

$K a t G P_{B} \quad$ GAGGCGGAGGTCATC(TACTGG)GGTCTATGTCCTGATTGTTC(GATATC)CGACAC[T]TCGCGATCAC

$K a t G P_{B} \quad$ ACGAGGCGGAGGTCA(TCTACT)GGGGTCTATGTCCTGATTGTTC(GATATC)CGACAC[T]TCGCGATCAC

Kat $G P_{C} \quad$ CCTGATTGTTCGATA(TCCGAC)ACTTCGCGATCACATCCGTGAT(CACAGC)CCGATAA[C]ACCAACTCCT

Kat $G P_{C} \quad$ TTCGATATCCGACAC(TTCGCG)ATCACATCCGTGAT(CACAGC)CCGATAA[C]ACCAACTCCT

PurL CGGCTTGTCCGTTTC(CACGCG)GCCGCAGCGCGATGGGGCCTAGC(TAGACT)GCCTCC[G]TGATGTCTCC
\end{abstract}

\footnotetext{
${ }^{1}$ Consensus regions are shown in bold letters and transcription start sites are shown in a square bracket.
} 


\begin{abstract}
PurC ATCTCATACCAGAGA(TACCAG)CACAGGGCGCCGTCGTGCGGCGGA(TAGgCT)GGCGTG[A]TGCGCCCCGC GroE CAGGAAGCAAGGGGGCG(CCCTTG)AGTGCTAGCACTCTCATGT(ATAGAG)TGCTAGATGGCAATCGGCTA GroE CAGGAAGCAAGGGGG(CGCCCTTG)AGTGCTAGCAC(TCTCATGTATAGAG)TGCTAGATGGCAATCGGCTA AhpC TGTGATATATCACCT(TTGCCT)GACAGCGACTTCACGG(TACGAT)GGAATGTCGTAACCAAATGC $32 \mathrm{kDa}$ ACATGCATGGATGCG(TTGAGA)TGAGGATGAGGGAAGC(AAGAAT)GCAGCTTGTTGACAGGGTTC $10 \mathrm{kDa}$ $10 \mathrm{kDa}$ $10 \mathrm{kDa}$ $65 k D a$ Mpt64 MetA RpsL $38 k D a$ Ppgk AAGCAAGGGGCGCCC(TTGAGT)GTCAGCACTCTCATGTA(TAGAGT)GCTAGATGGCAATCGGCTAA AAGCAAGGGGCGCCC(TTGAGT)GTCAGCACTCTCATG(TATAGA)GTGCTAGATGGCAATCGGCT AAGCAAGGGGCGCCC(TTGAGT)GTCAGCAC(TCTCAT)GTATAGAGTGCTAGATGGCA GCGTAAGTAGCGGGG(TTGCCG)TCACCCGGTGACCCCCG(TTTCAT)CCCCGATCCGGAGGAATCAC GAGTCTGGTCAGGCA(TCGTCG)TCAGCAGCGCGATGCCC(TATGTT)TGTCGTCGACTCAGATATCG TCCGGCCCCCGCGAT(TTGGCG)AGCTTCGTGCGTGTTCGG(TAGCCT)GGCATTTACCGACGCGGGGT GCCGCAACGCCCGCT(TTGACC)TGCCAGACTGGCGGCGGG(TATTGT)GGTTGCTCGTGCCTGGCGGC CGTCGCCGGACTGTCGGGGGACGTCAAGGACGCCAAGCGCG(GAAATT)GAAGAGCACAGAAAGGTATG CGGGCCGCAGTTTAAGGTGAGGGTCATCCACGTCTCGCCGAGGAGATTCGATGACCAGCAC
\end{abstract}

M. bovis $B C G$

Hsp60 P2 CGGTGCGGGGCTTCTTGCACTCGGCATAGGCGAGTGC(TAAGAA)TAACGTT[G]

RRNA TGACCGAACCTGGTC(TTGACT)CCATTGCCGGATTTG(TATTAG)ACTGGCAGGGTTGCCCCGAA AhpC TGTGATATATCACCT(TTGCCT)GACAGCGACTTCACGG(TACGAT)GGAATGTCGCAACCAAATGC

$23 K$

$m p b 64$ GAGTCTGGTCAGGCA(TCGTCG)TCAGCAGCGCGATGCCC(TATGTT)TGTCGTCGACTCAGATATCG GAGTCTGGTCAGGCA(TCGTCG)TCAGCAGCGCGATGCCC(TATGTT)TGTCGTCGACTCAGATATCG $18 K$

$64 K$

rpsL $\operatorname{mpb} 70$ alpha TGGCGTCCGAAACAC(TTGAGG)TGCGGCCCAGCAAGGGGC(TACAGG)TTTTTTCCTTCACCTACGGA GCGTAAGTAGCGGGG(TTGCCG)TCACCCGGTGACCCCCGG(TTTCAT)CCCCGATCCGGAGGAATCAC GCCGCAACGCCCGCT(TTGACC)TGCCAGACTGGCGGCGGG(TATTGT)GGTTGCTCGTGCCTGGCGGC TGGCGTCCGAAACAC(TTGAGG)TGCGGCCCAGCAAGGGGC(TACAGG)TTTTTTCCTTCACCTACGGA CGACTTTCGCCCGAA(TCGACA)TTTGGCCTCCACACACGG(TATGTT)CTGGCCCGAGCACACGACGA

M. leprae $16 S$ rRNA $18 \mathrm{Kda}$ $18 \mathrm{Kda}$ $28-k D a$ groE1 $65 \mathrm{kd}$ $36 k$ SOD rpsL TAGTCAACCCGGGAC(TTGACT)CCTCTGCTGGATCTGT(ATTAAT)CTGGCTG[G]GTTGCCGAAG CTTGTCTATCACAAC(TTGCAT)CAATATATCGACCAGTG(CTATAT)CAAATCTA[T]GTAGTCAGGA CTTGTCTATCACAAC(TTGCAT)CAATATATCGACCAGTGC(TATATC)AAATCTA[T]GTAGTCAGGA TCAATATAACCACTC(TGGTCA)CACTAACCATACTCG(TACCAT)CAACCGTGTGGGGCTAATCC AGCAGCGGGCCGGCC(TTGAGT)GCTAGCACTCGCGTGTA(TAGAGT)GCTAGATGGCAGTCGGCCAG GAATTCCGGAA(TTGCAC)TCGCCTTAGGGGAGTGC(TAAAAA)TGATCCTGGCACTCGCGATC GTTGGG(TTTCCT)CTCGGAGGGCGCACCGC(TACGTT)AGCGGGATG GG(TGGGCG)CGATCATGGCGCAGCGTT(GATTAT)GCTAGTCG CGCCGTTGGGTCGCT(TTGACC)TGCCCGAGCAGGGACGGG(TATTGT)GTTTCTCGTTCCTGACGGCT

M. smegmatis

alra GTCTGCGGCCTCTGG(GACAAT)GGGCGCC[G]GAGATTATGA

S4 AAGCCGAATCGAGACCTTTTGGGTTCGTACACACTTGCTT(TATAAG)CCTC[G]

S5 AACAAGATTCCGTTAATCGTGTCTGGTGGAGCTGGTGG(TAAGCT)TGATCC[G]

S6 CATCGATTTTAAATTTTGA(TAGAGT)GCAAATA[A]

S12 ACCTCGTTATGCTTCTGGCTATTTTTGATCAACTTT(TATACA)TGGGCGGT[T]

S14 TCAAGCACCCAAGCCAACATGGTTGTAGTAGTCGTTT(TACCAT)GTGTACC[T]

S16

S18

$S 19$

$S 21$

S30

S33

S35

S65

S69

S119

gyrB

recA

ask TCCACGCGAACCGCTTCGGCGTGCCCCGTTTTCCCTGT(TATAAT)ATCGGC[G] GATCATTGTCTTCTGTTGTCTTTCGTA(TAAAGT)TGTTACT[G] TTTGATGTAGCCAAAGGCTCTCACCACCTGAGCCATGA(TAGTAT)CCATCC[C] ACATGGCATTTTTCATTTAAAACAGGACTCAGGTGG(TATGGT)TGACATCG[A] GATCAGCTATGTTCTTCAGTAAAATTTCGGC(TATATG)TTGGT[G] GATCCGCTCTTCTTATGATGCCAGTTATGGTATC(TATGGT)TATC[G] AACTAAAGTATGTGCCGTAATTGACAGTGTTCTAGAT(TATGAT)GCTGCAT[C] GGCACAGCTCGAAGTTCTACTACATGGCTTGCTGAA(TCCAGT)CACATTAC[T] ATCACGATGTCTTCATGCTTGGCTTCAATGCTCCGGTC(TACAAT)CAGTTC[A] GATCAAGAAGCCAATGATTTGT(TAAACG)CAATTAAT[G]

CAGAATCGGTGCTGT(CGCTAT)CTCGCGG(TAGACT)GGACGAC[G]GATCTCAGGC AGAGTTCGACCGGAC(TTGTCG)GTGGTCTGC(TCTAAC)GTCACGGCC[A]ACCGATCGGA GT(TTGCCC)GCCGCGGCGCCC(CACGAT) GAACCGC[A]CGGGCTGACG acetamidase GGCCGGCGTTCACCC(TTGACT)TTTATTTTCATCTGGA(TATATT)TCGGGT[G]AATGGAAAGG 
$r r n B$ CTCTGACCTGGGGAT(TTGACT)CCCAGTTTCCAAGGACG(TAACTT)ATTCCAG[G]TCAGAGCGAC $r r n A P 1$ GAAAACCTGGTCAGC(CTCGGA)GCCGAGATCGAGAGAG(TAAGCT)CGTAG[G]AAGCAAGACC $r r n A P 2 \quad$ CTCTGACCAGGCGAT(TTGCAA)TCGCGACGAACCTCGTAT(TATCTT)TATGAA[G]TCGCCGCGGA $r r n A P 3$ CCGGGCCAGAGCGAC(TTGACA)AGCCAGCCGAGATCGTAC(TAAGCT)GGCGAG[G]TTGCCTCAGA $r r n A$ PCL1 CCGGTCCAGAGCGAC(TTGACA)AGCCAGACAAAGCAGTAT(TAAGCT)GGCAGG[G]TTGCCCCAAA $r p s L \quad$ CCGCCGTGCACGAGT(TTGTTT)CGTCGCGGTCGCCCCTGG(TATTGT)GGTGGATC[G]TGCCTGGCCC $r p s L \quad$ CGTGCACGAGTTTGT(TTCGTC)GCGGTCGCCCCTGGTAT(TGTGGT)GGATC[G]TGCCTGGCCCGAAA ahp C TGTGATATATCACCT(TTGCCT)GACAGCGACTTCACGG(CACGAT)GGAATGTCGCAACCAAATGC

M. paratuberculosis

pAJB303 GACGACGAGGGCGG(TGGCGT)CGCCGGTGTAGCCGAA(CGGCAC)GTGCGCG[T]AGGCCCAGAT pAJB86 $p A J B 125$ pAJB300 pJB305 pAJB304 $P_{A N}$ pAJB73 pAJB301 CCACCTTACTCCCGA(TGACGT)TGCACGGCTGGGATTAA(CGGTCC)GCGTGC[T]CCAGGAGACA GCAACGAGCGCATCA(TTAAAG)ATCGANGGCGCCGGGNT(CATGTC)CCTTCAC[C]CCGCCCAGCT TCGAGTTCAAGACCC(TGACGC)TGGCCGACCTCGGCGCG(CAGCCG)ACCGCGC[A]GCGGTGCACG ATCCGGACGGGCAGT(TGTTGG)AGTTTCTGTCGGACGGT(TGGTTG)GCGGCAT[T]TCCGGCGAGG CACCAGGTACACGCC(AAGGAC)AACGGCCGTATCCGGTA(CCAACG)GGTGTGC[G]AGCTGGACGG CTGGTGAAGGGTGAA(TCGACA)GGTACACACAGCCGCCA(TACACT)TCGCTTC[A]TGCCCTTACG GATCGGTG(TGCCGC)TTGAACCGGCCCAGCTCCCG(CTCCAG)GGTGACG[T]GCTCGAGCTC

M. fortuitum repA GAGCTCGTGTCGGACCATACACCGGTGATTAATCGTGG(TCTACT)ACCAAG[C]

rrnA $P C L 1$ CCAGGATGATGCAAC(TTGACT)TGCCGGCAAGATTCGAAT(TAAGCT)GGCGGG[G]TTGCCCCAAA rrnA $P 1$ $\operatorname{rrnAP} P 2 a$ $\operatorname{rrnAP} P 2 b$ rrnA $P 3$ GAAAACCTGTTGAGC(CTCGGA)GCCGAGATCGAAAGAG(TAGGGT)CGTAAACAGCAGTCCGGGCC CGCTGACCAGCCGAT(TTGACC)TTGTAGGCAGGCCCGCGC(TAATCT)TTTGAAGTCGCGCGGAGCGG CCGGGCCAGAGCGAC(TTGACA)AGCCAGCCGAGATCGTAC(TAAGCT)GGCGAGGTTGCCTCAGACCG CAGGATGATGCAACT(TGACTT)GCCGGCAAGATTCGAATT(AAGCTG)GCGGGGTTGCCCCAAAACAG

M. phlei rrnA PCL1 ACTGGGGACGAGGTC(TTGACG)CCCCTGATCAGATCGGTA(TAGACT)GGCAGG[G]TTGCCCGAAA rrnA $P 1$ $\operatorname{rrnAP2}$ $\operatorname{rrnAP} P$ GAGAACCTCCGCAGT(CTCGGC)GCCGAGATCGAGAGGG(TCGCCT)GAAACATGCCGTTTACCTGC AGGGGACCCCCCTTT(TTGACT)CCGCTCAGACGTGGGC(TATTCT)TCTAACCACAAGCCCAACGC CTGGGGACGAGGTCT(TGACGC)CCCTGATCAGATCGGTAT(AGACTG)GCAGGGTTGCCCGAAAGCAA

Mycobacteriophage $I 3$

pKGR25 CCTGTACACCCTCGC(TGCACT)CGCCGAGGACAAG(CACTAT)CGCCCCGACGTCCCGGCCTGG pKGR9 pKGR38 ACCACGAGCACCCGG(TCGTCA)GGACTGCGACACTCGA(TGTTGT)AGACGCACTGGTGCAGCATG ORF1 ATCTGGTCGACCTGC(TCGACG)AGGTCGATCATCTTCT(TCATCT)CGCCGAACGGGATGCCCTGG

ORF2 ACCTCATGGAGCACT(TCGAGG)TCACTGAGCACGCCCA(CGAACT)ACGAGAGGCCGTGGGACTGG TACTTTTTGTACCGT(TCGACA)CCAGCGGTTTCCGCTTCCTTGC(CAATCT)CCTGCAAACAAACCACAATG ACACAGACCAGGAGC(TCGACA)TGACCGCCACCGCCCCCTACAGCG(TCATCT)GGTTCGAAGGCACCCCGGAT

pKGR1

Mycobacteriophage L5

$71 P 2$ TACCTGTCACAAGGT(TTGCTA)CCGAGTGGGGCAGGCCGC(TACATT)TACGACC[G]CGTAACGCCA

$71 P_{\text {left }}$ TTtGCGATtAGGGC(TTGACA)GCCACCCGGCCAGTAGTG(CATTCT)TGTGTC[A]CCGCAGCAGC

$71 P 1 \quad$ ACAACTGAATATGGT(TCCGCA)GACGCAACTAAATTAGGGG(TATCCT)TGACA[G]GCACCAACAT

M. avium

Avi-3

pLR7

GCCGGCGATCGTGGG(CTGATA)AGTCTTATCGGGCATAC(TATAAG)TGTAGTGGGAAATATCACCT AGCCTTGTTGGCGGC(CAACTG)CCGGACGATCGCGGCGGC(CATCGT)CCTCGAGCTCGGCCCCGTGC

M. neoaurum

rrnA PCL1 GCGAGACAGAGAAGC(TTGACT)CGCCAGACAAGATAGTT(TAAGCT)GGCAGG[G]TTGCCCCGAA $\operatorname{rrnAP1}$

$\operatorname{rrnAP} P$ GAAAACCTGGTCAGC(TTGGGC)GCCGGGATCGAGCGAG(TACACT)CGTAAGAGACCGGTCGAGTG $\operatorname{rrnA} P 2$ GCGAGACAGAGAAGC(TTGACT)CGCCAGACAAGATAGTT(TAAGCT)GGCAGGGTTGCCCCGAAACG CTCTGACCAGCGGAT(TTGACT)TCCGAAGGCACAAAGTTC(TAATCT)TTTGAAGTCGCCGCGGGGAG

M. abscessus

$r r n A P 4$ GCCAAAACCGGGAAT(TTGACT)CAGGTTCACGAACTTGA(TACGGT)TTCCGA[G]CGCCCGAAAG $r r n A 1$ GGCGGGTCTAGTGGC(GGACGG)CGTCACAGAGGTATACGA(TGTGTT)TCATATCG[A]CCGCGGTTAC 
$\begin{array}{ll}r r n A P C L 1 & \text { GCCCCCGACCCGAAG(TTGACT)CAAGTTCATTGGACTTGG(TACAGT)GGTCGG[G]TTGCCCTGAA } \\ r r n A P 2 & \text { GCCAAAACCGGGAAT(TTGACT)CAAGTTCACCGAACT TGA (TACGGT)TTCC[A]AGTCGCTCGG } \\ r r n A P 3 & \text { GCCAAAACCGGGAAT(TTGACT)CAAGTTCACCGAACT } \underline{T G A} \text { (TACGGT)TTCCAA[G]TCGCTCGGAA }\end{array}$

M. chelonae

$r r n A P 2$ CCAAAACCCGGAGTT(TGACTC)AAGTTCACCGAACTTGA(TCGGTT)CCCGG[G]CCGCTTACAA

$r r n A P 1$ GGCGGGGTTAGTGGC(GGATGG)CGTCACCGAGGTATACGA(TGTGTT)TCATATC[G]ACCGCGGTTA

$r r n A P C L 1$ CCCCAGAACCCGAAG(TTGACT)CAAGTTCATTGGACTTGG(TACAGT)GGTCGG[G]TTGCCCTGAA

$r r n A P 3 \quad$ GCCAAAACCGGGAAT(TTGACT)CAAGTTCACCGAACTTGA (TCGGTT)TCCCA[G]CCGCCCGAAA

$r r n A P 4$ GCCAAAACCGGGAAT(TTGACT)CAAGTTCACCGAACTTGA (TACGGT)TTCCGA[G]CCGCCCGAAA

\section{References}

[1] T.M. Shinnick, The 65-kilodalton antigen of Mycobacterium tuberculosis, J. Bacteriol. 169 (1987) 1080-1088.

[2] J.E. Thole, H.G. Dauwerse, P.K. Das, D.G. Groothuis, L.M. Schouls, E.J.D. van, Cloning of Mycobacterium bovis BCG DNA and expression of antigens in Escherichia coli, Infect. Immun 50 (1985) 800-806.

[3] V. Mehra, D. Sweetser, R.A. Young, Efficient mapping of protein antigenic determinants, Proc. Natl Acad. Sci. USA 83 (1986) 7013-7017.

[4] L. Kremer, A. Baulard, J. Estaquier, J. Content, A. Capron, C. Locht, Analysis of the Mycobacterium tuberculosis $85 \mathrm{~A}$ antigen promoter region, J. Bacteriol. 177 (1995) 642-653.

[5] F. Movahedzadeh, M.J. Colston, E.O. Davis, Determination of DNA sequences required for regulated $\mathrm{Myco}$ bacterium tuberculosis RecA expression in response to DNA-damaging agents suggests that two modes of regulation exist, J. Bacteriol. 179 (1997) 3509-3518.

[6] M.D. Bashyam, D. Kaushal, S.K. Das Gupta, A.K. Tyagi, A study of mycobacterial transcriptional apparatus: identification of novel features in promoter elements, J. Bacteriol. 178 (1996) 4847-4853.

[7] M.A. Mulder, H. Zappe, L.M. Steyn, Mycobacterial promoters, Tubercle. Lung Dis 78 (1997) 211-223.

[8] C.B. Hoopes, W.R. McClure, Strategies in regulation of transcription initiation, in: F.C. Neidhart (Ed.), Escherichia coli and Salmonella typhimurium, American Society of Microbiology, Washington DC, 1987, pp. $1231-1239$

[9] J. Ramstein, R. Lavery, Energetic coupling between DNA bending and base pair opening, Proc. Natl. Acad. Sci. USA 85 (1988) 7231-7235.

[10] J.D. Parvin, R.J. McCormick, P.A. Sharp, D.E. Fisher, Pre-bending of a promoter sequence enhances affinity for the TATA-binding factor, Nature 373 (1995) 724-727.

[11] C.M. Collis, P.L. Molloy, G.W. Both, H.R. Drew, Influence of the sequence-dependent flexure of DNA on transcription in E. coli, Nucl. Acids Res 17 (1989) 9447-9468.

[12] R.R. Plaskon, R.M. Wartell, Sequence distributions associated with DNA curvature are found upstream of strong E. coli promoters, Nucl. Acids Res 15 (1987) 785-796.

[13] L. Bracco, D. Kotlarz, A. Kolb, S. Diekmann, H. Buc, Synthetic curved DNA sequences can act as transcriptional activators in Escherichia coli, EMBO J. 8 (1989) 4289-4296.

[14] T. Ohyama, M. Nagumo, Y. Hirota, S. Sakuma, Alteration of the curved helical structure located in the upstream region of the beta-lactamase promoter of plasmid pUC19 and its effect on transcription, Nucl. Acids Res 20 (1992) 1617-1622.

[15] T. Gaal, L. Rao, S. Estrem, J. Yang, R. Wartell, R. Gourse, Localization of the intrinsically bent DNA region upstream of the E.coli $\mathrm{rrnB}$ P1 promoter, Nucl. Acids Res 22 (1994) 2344-2350.

[16] M. Engelhorn, A. Geiselmann, Maximal transcriptional activation by the IHF protein of Escherichia coli depends on optimal DNA bending by the activator, Mol. Microbiol 30 (1998) 431-441.

[17] S.E. Aiyar, R.L. Gourse, W. Ross, Upstream A-tracts increase bacterial promoter activity through interactions with the RNA polymerase alpha subunit, Proc. Natl. Acad. Sci. USA 95 (1998) 14652-14657.

[18] E.N. Trifonov, Curved DNA, CRC Crit. Rev. Biochem. 19 (1985) 89-106.

[19] P.J. Hagerman, Sequence-directed curvature of DNA, Annu. Rev. Biochem 59 (1990) 755-781.

[20] A. Klug, A. Jack, M.A. Viswamitra, O. Kennard, A. Shakked, T.A. Steitz, A hypothesis on a specific sequence-dependent conformation of DNA and its relation to the binding of the lac-repressor protein, J. Mol. Biol. 131 (1979) 669-680.

[21] R.E. Dickerson, H.R. Drew, Structure of a B-DNA dodecamer. II. Influence of base sequence on helix structure, J. Mol. Biol. 149 (1981) 761-786.

[22] P.J. Hagerman, Evidence for the existence of stable curvature of DNA in solution, Proc. Natl. Acad. Sci. USA 81 (1984) 4632-4636.

[23] S.C. Satchwell, H.R. Drew, A.A. Travers, Sequence periodicities in chicken nucleosome core DNA, J. Mol. Biol. 191 (1986) 659-675.

[24] C.R. Calladine, H.R. Drew, M.J. McCall, The intrinsic curvature of DNA in solution, J. Mol. Biol. 201 (1988) 127-137. 
[25] A. Bolshoy, P. McNamara, R.E. Harrington, E.N. Trifonov, Curved DNA without A-A: experimental estimation of all 16 DNA wedge angles, Proc. Natl. Acad. Sci. USA 88 (1991) 2312-2316.

[26] C.A. Hunter, Sequence-dependent DNA structure. The role of base stacking interactions, J. Mol. Biol. 230 (1993) 1025-1054.

[27] C.A. Hunter, Sequence-dependent DNA structure, Bioessays 18 (1996) 157-162.

[28] D.S. Goodsell, R.E. Dickerson, Bending and curvature calculations in B-DNA, Nucl. Acids Res 22 (1994) 5497-5503.

[29] I. Brukner, R. Sanchez, D. Suck, S. Pongor, Sequencedependent bending propensity of DNA as revealed by DNase I: parameters for trinucleotides, EMBO J. 14 (1995) 1812-1818.

[30] M.A. EI Hassan, C.R. Calladine, Propeller-twisting of base-pairs and the conformational mobility of dinucleotide steps in DNA, J. Mol. Biol. 259 (1996) 95-103.

[31] C. Anselmi, G. Bocchinfuso, P. De Santis, M. Savino, Dual role of DNA intrinsic curvature and flexibility in determining nucleosome stability, J. Mol. Biol. 286 (1999) 1293-1301.

[32] M. Dlakic, R.E. Harrington, The effects of sequence context on DNA curvature, Proc. Natl. Acad. Sci. USA 93 (1996) 3847-3852.
[33] P. De Santis, A. Palleschi, M. Savino, A. Scipioni, A theoretical model of DNA curvature, Biophys. Chem 32 (1988) 305-317.

[34] C.R. Calladine, Mechanics of sequence-dependent stacking of bases in B-DNA, J. Mol. Biol. 161 (1982) 343-352.

[35] R.E. Dickerson, Base sequence and helix structure variation in B and A DNA, J. Mol. Biol. 166 (1983) 419-441.

[36] E.S. Shpigelman, E.N. Trifonov, A. Bolshoy, curvature: software for the analysis of curved DNA, Comp. Appl. Biosci 9 (1993) 435-440.

[37] E.N. Trifonov, L.E. Ulanovsky, Inherently curved DNA and its structural elements, in: R.D. Wells, S.C. Harvey (Eds.), Unusual DNA Structures, Springer-Verlag, Berlin, 1987, pp. 173-187.

[38] P. De Santis, A. Palleschi, M. Savino, A. Scipioni, Validity of the nearest-neighbor approximation in the evaluation of the electrophoretic manifestations of DNA curvature, Biochemistry 29 (1990) 9269-9273.

[39] P. De Santis, A. Palleschi, M. Savino, A. Scipioni, Theoretical prediction of the gel electrophoretic retardation changes due to point mutations in a tract of SV40 DNA, Biophys. Chem 42 (1992) 147-152.

[40] T.M. Nair, B.D. Kulkarni, On the consensus structure within the E. coli promoters, Biophys. Chem 48 (1994) 383-393. 